\section{Pacific Northwest}

National Laboratory

Operated by Battelle for the

U.S. Department of Energy

\title{
Hanford Site Environmental Surveillance Master Sampling Schedule
}

LE Bisping

January 2002

Prepared for the U.S. Department of Energy under Contract DE-AC06-76RL01830 


\title{
DISCLAIMER
}

This report was prepared as an account of work sponsored by an agency of the United States Government. Reference herein to any specific commercial product, process, or service by trade name, trademark, manufacturer, or otherwise does not necessarily constitute or imply its endorsement, recommendation, or favoring by the United States Government or any agency thereof, or Battelle Memorial Institute.

\author{
PACIFIC NORTHWEST NATIONAL LABORATORY \\ operated by \\ BATTELLE \\ for the \\ UNITED STATES DEPARTMENT OF ENERGY \\ under Contract DE-AC06-76RL01830
}

Printed in the United States of America

Available to DOE and DOE contractors from the

Office of Scientific and Technical Information, P.O. Box 62, Oak Ridge, TN 37831;

prices available from (615) 576-8401.

Available to the public from the National Technical Information Service,

U.S. Department of Commerce, 5285 Port Royal Rd., Springfield, VA 22161

This document was printed on recycled paper. 
PNNL-13749

\title{
HANFORD SITE ENVIRONMENTAL SURVEILLANCE MASTER SAMPLING SCHEDULE
}

\author{
L. E. Bisping
}

January 2002

Prepared for the U.S. Department of Energy

under Contract DE-AC06-76RL01830

Pacific Northwest National Laboratory

Richland, Washington 99352 


\section{SUMMARY}

Environmental surveillance of the Hanford Site and surrounding areas is conducted by the Pacific Northwest National Laboratory (PNNL) ${ }^{(a)}$ for the U.S. Department of Energy (DOE). Sampling is conducted to evaluate levels of radioactive and nonradioactive pollutants in the Hanford environs, as required in DOE Order 5400.1, “General Environmental Protection Program,” and DOE Order 5400.5, "Radiation Protection of the Public and the Environment." The sampling design is described in the Environmental Monitoring Plan, United States Department of Energy, Richland Operations Office, DOE/RL-91-50, Rev.3, U.S. Department of Energy, Richland, Washington.

This document contains the CY 2002 schedules for the routine collection of samples for the Surface Environmental Surveillance Project (SESP) and Drinking Water Monitoring Project. Each section includes sampling locations, sample types, and analyses to be performed. In some cases, samples are scheduled on a rotating basis and may not be collected in 2002 in which case the anticipated year for collection is provided. In addition, a map showing approximate sampling locations is included for each media scheduled for collection in 2002.

\section{SESP SAMPLING}

The SESP is a multimedia environmental surveillance effort to measure the concentrations of radionuclides and chemicals in environmental media and assess the integrated effects of these materials on the environment and the public. Project staff collect samples of air, surface water, agricultural products, wildlife, and sediments. In addition, soil and natural vegetation samples are collected approximately every 5 years. Analytical capabilities include the measurement of radionuclides at very low environmental concentrations and, in selected media, nonradiological chemicals including metals, anions, and volatile organic compounds. In addition, the project includes the capability to measure ambient external radiation.

\section{DRINKING WATER MONITORING PROJECT SAMPLING}

The responsibility for monitoring onsite drinking water falls outside the scope of the SESP. The operator of the onsite drinking water systems (Fluor Hanford, Inc.) is responsible for monitoring drinking water quality as defined in the National Drinking Water Standards and Washington Administrative Code (WAC) 246-290. PNNL conducts radiological monitoring of onsite drinking water for Fluor Hanford concurrent with the SESP to promote efficiency and consistency, utilize expertise developed over the

(a) Pacific Northwest National Laboratory is operated by Battelle Memorial Institute for the U.S. Department of Energy under Contract DE-AC06-76RL01830. 
years, and reduce costs associated with management, procedure development, analytical contracting, data management, quality control, and reporting.

\section{DATA MANAGEMENT}

The Hanford Environmental Information System (HEIS) database is used as a repository for data gathered during environmental surveillance activities at the Hanford Site. For ease in retrieving these data from the HEIS database, the location names in this document reflect the exact location names used in the HEIS.

\section{SCHEDULED CHANGES}

This schedule is subject to modification during the year in response to changes in site operations, program requirements, and the nature of the observed results. Operational limitations such as weather, mechanical failures, sample availability, etc., may also impact scheduled sampling. Therefore, this document may not be an accurate record of samples collected during the year.

\section{COSAMPLES}

Samples that are cosampled and analyzed by both PNNL and the Washington State Department of Health (DOH) are indicated in the schedule as are samples that are cosampled and analyzed by both PNNL and the U.S. Food and Drug Administration (FDA).

\section{ADDITIONAL INFORMATION}

Questions relating to the content of this document can be directed to T. M. Poston, Manager, Surface Environmental Surveillance Project, (509) 376-5678 or R. W. (Bill) Hanf, Manager, Drinking Water Monitoring Project, (509) 376-8264. 


\section{CONTENTS}

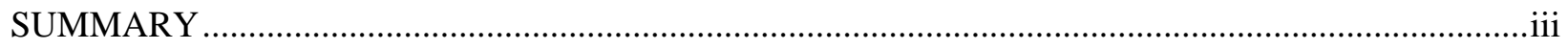

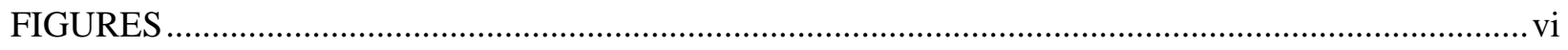

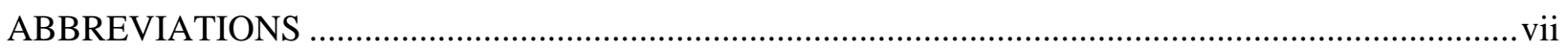

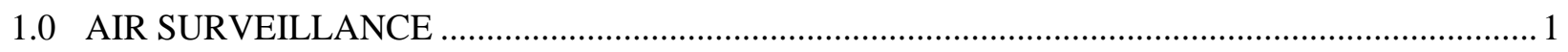

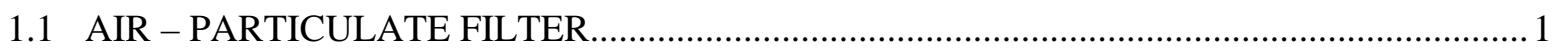

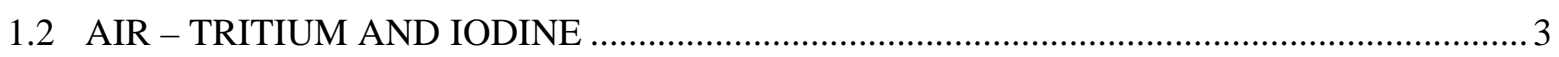

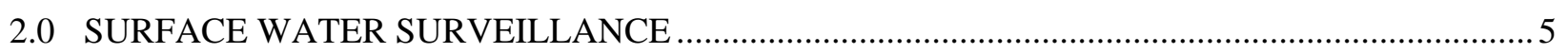

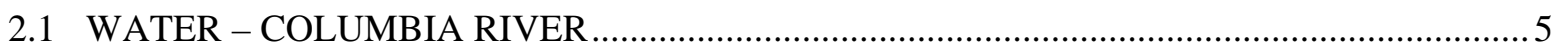

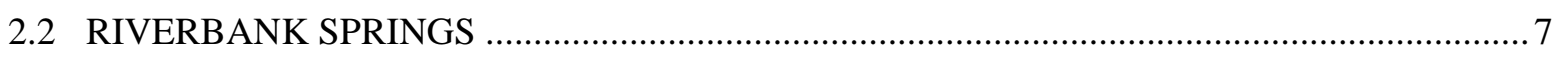

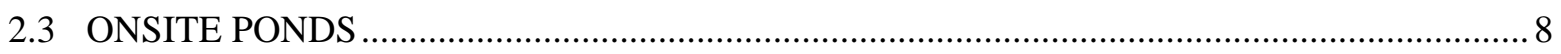

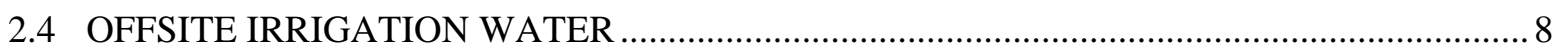

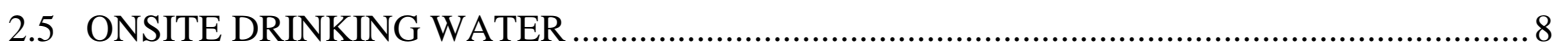

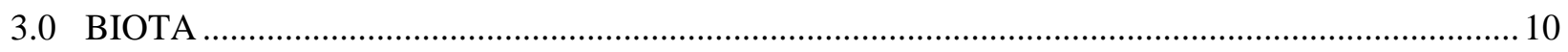

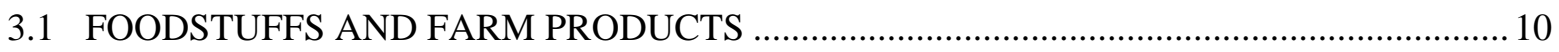

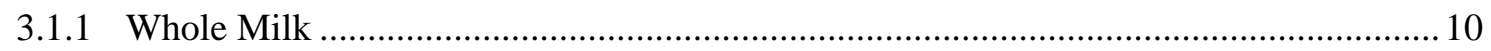

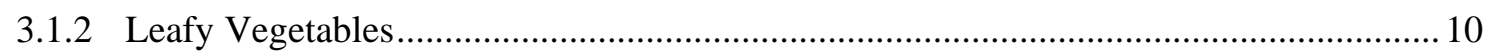

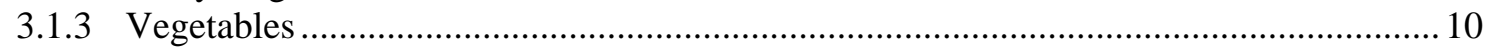

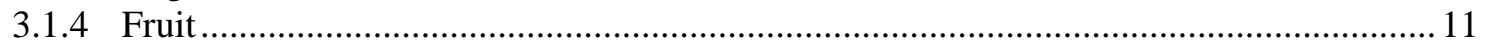

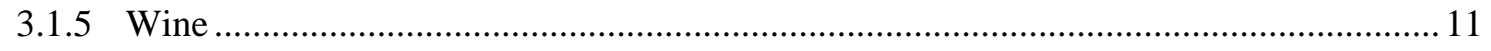

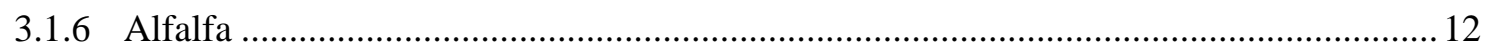

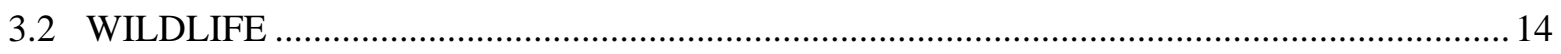

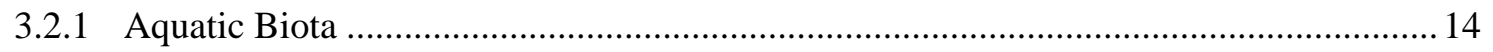

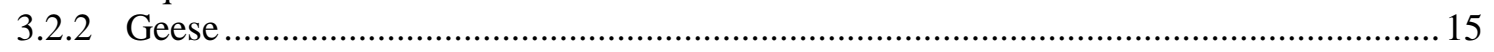

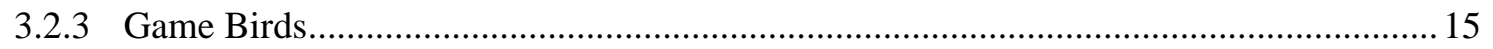

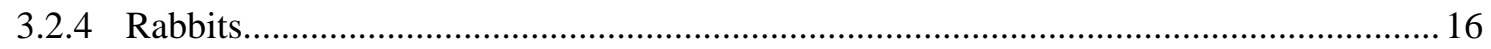

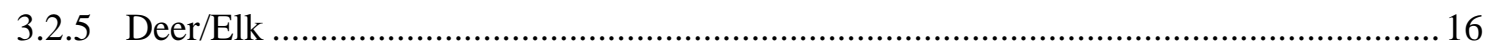

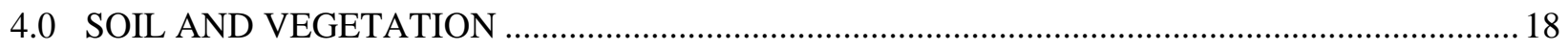

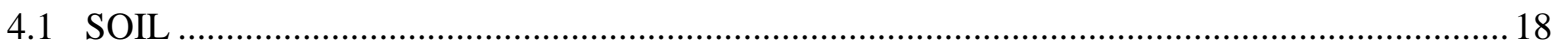

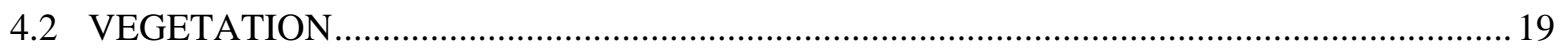

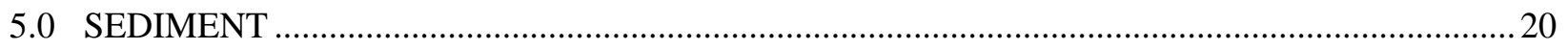

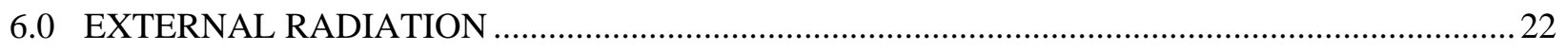

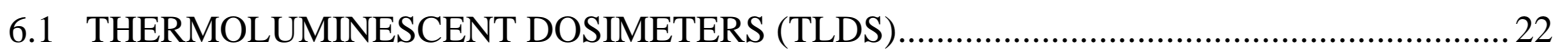

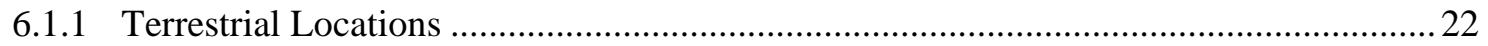

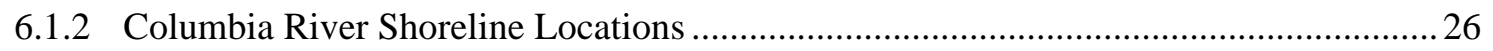

6.2 COLUMBIA RIVER SHORELINE RADIATION SURVEYS............................................ 26 


\section{FIGURES}

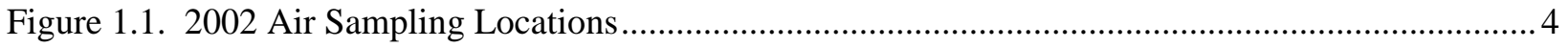

Figure 2.1. 2002 Surface Water and Drinking Water Sampling Locations ............................................. 9

Figure 3.1. 2002 Food and Farm Product Sampling Locations ........................................................... 13

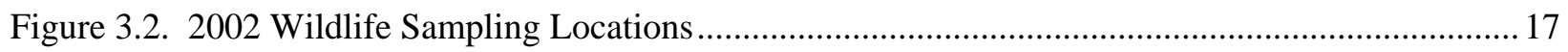

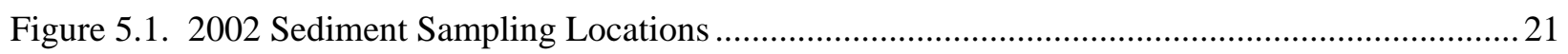

Figure 6.1. 2002 Thermoluminescent Dosimeter (TLD) Locations on the Hanford Site .........................24

Figure 6.2. 2002 Thermoluminescent Dosimeter (TLD) Locations for Perimeter,

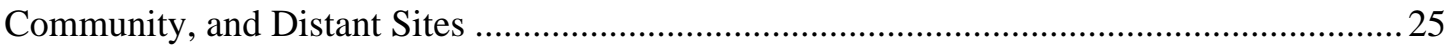

Figure 6.3. 2002 Thermoluminescent Dosimeter (TLD) Locations on the

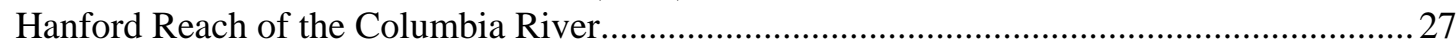




\section{ABBREVIATIONS}

\section{FREQUENCY SYMBOLS USED}

$\begin{array}{ll}\text { A } & \text { annually } \\ \text { BE } & \text { biennial (every 2 years) } \\ \text { BW } & \text { biweekly (every 2 weeks) } \\ \text { M } & \text { monthly } \\ \text { M Comp. } & \text { monthly composite } \\ \text { Q } & \text { quarterly } \\ \text { Q Comp. } & \text { quarterly composite } \\ \text { SA } & \text { semiannually } \\ \text { TE } & \text { triennial (every 3 years) }\end{array}$

ANALYTICAL SYMBOLS USED

Generally, standard element, chemical, and isotope designations are used to indicate the analyses performed. Other analytical designations used are:

\begin{tabular}{|c|c|}
\hline Alpha & gross alpha activity of sample \\
\hline Anions & major anions-generally chloride, fluoride, nitrate, nitrite, sulfate \\
\hline Beta & gross beta activity of sample \\
\hline Gamma Scan & $\begin{array}{l}\text { analysis of photon energy spectrum for individual photon-emitting } \\
\text { radionuclides }\end{array}$ \\
\hline HTO & tritiated water $\left({ }^{3} \mathrm{H}^{1} \mathrm{H}^{16} \mathrm{O}\right)$ \\
\hline ICP-u, ICP-3 & $\begin{array}{l}\text { major metals by inductively coupled plasma spectrometry - samples unfiltered } \\
\text { unless otherwise noted }\end{array}$ \\
\hline Lo ${ }^{3} \mathrm{H}$ & analytical procedure includes electrolytic enrichment \\
\hline $\mathrm{Pu}$ & Isotopic plutonium $\left({ }^{238} \mathrm{Pu},{ }^{239 / 240} \mathrm{Pu}\right)$ \\
\hline SEM/AVS & Simultaneously Extracted Metals/Acid Volatile Sulfide \\
\hline TOC & Total Organic Carbon \\
\hline $\mathrm{U}$ & Isotopic uranium $\left({ }^{234} \mathrm{U},{ }^{235} \mathrm{U},{ }^{238} \mathrm{U}\right)$ \\
\hline VOA & Volatile Organic Compounds \\
\hline
\end{tabular}

\section{INSTRUMENT SYMBOLS USED}

$\begin{array}{ll}\text { BICRON } & \text { Microrem meter } \\ \text { GM } & \text { Geiger-Müeller counter } \\ \text { PIC } & \text { Pressurized ionization chamber }\end{array}$




\subsection{AIR SURVEILLANCE}

\subsection{AIR - PARTICULATE FILTER}

Location

Location

Number $^{(a)}$
Individual Samples

Fre-

quency ples

Analyses $\frac{\text { Composited Samples }}{\text { Fre- }}$

quency
Analyses

$\underline{\text { Onsite }}$

\begin{tabular}{|c|c|c|c|c|c|c|c|}
\hline 100 K Area & & BW & Beta , Alpha & \multirow{3}{*}{\multicolumn{2}{|c|}{100 Areas }} & & \\
\hline 100 N-1325 Crib & 2 & BW & Beta, Alpha & & & $\mathrm{Q}$ & ${ }^{90} \mathrm{Sr}, \mathrm{Pu}, \mathrm{Gamma}$ Scan \\
\hline 100 D Area & 3 & BW & Beta, Alpha & & & & \\
\hline $\begin{array}{l}100 \text { F Met Tower } \\
\text { Hanford Townsite }\end{array}$ & $\begin{array}{l}4 \\
5\end{array}$ & $\begin{array}{l}\text { BW } \\
\text { BW }\end{array}$ & $\begin{array}{l}\text { Beta, Alpha } \\
\text { Beta, Alpha }\end{array}$ & \multicolumn{2}{|l|}{ Hanford Townsite } & Q & ${ }^{90} \mathrm{Sr}, \mathrm{Pu}, \mathrm{Gamma}$ Scan \\
\hline $\mathrm{N}$ of $200 \mathrm{E}$ & 6 & BW & Beta, Alpha & \multicolumn{2}{|l|}{$\mathrm{N}$ of $200 \mathrm{E}$} & $\mathrm{Q}$ & Gamma Scan \\
\hline E of $200 \mathrm{E}$ & 7 & BW & Beta, Alpha & \multicolumn{2}{|l|}{$\mathrm{E}$ of $200 \mathrm{E}$} & Q & ${ }^{90} \mathrm{Sr}, \mathrm{Pu}, \mathrm{U}$, Gamma Scan \\
\hline $\begin{array}{l}200 \mathrm{ESE}^{(\mathrm{b})} \\
S \text { of } 200 \mathrm{E}\end{array}$ & $\begin{array}{l}8 \\
9\end{array}$ & $\begin{array}{l}\text { BW } \\
\text { BW }\end{array}$ & $\begin{array}{l}\text { Beta, Alpha } \\
\text { Beta, Alpha }\end{array}$ & \multicolumn{2}{|l|}{200 E Area $^{(b)}$} & $\mathrm{Q}$ & ${ }^{90} \mathrm{Sr}, \mathrm{Pu}, \mathrm{U}$, Gamma Scan \\
\hline B Pond & 10 & BW & Beta, Alpha & \multicolumn{2}{|l|}{ B Pond } & $\mathrm{Q}$ & ${ }^{90} \mathrm{Sr}, \mathrm{Pu}, \mathrm{U}$, Gamma Scan \\
\hline $\begin{array}{l}\text { Army Loop Camp } \\
200 \text { Tel. Exchange } \\
\text { SW of B/C Cribs }\end{array}$ & $\begin{array}{l}11 \\
12 \\
13\end{array}$ & $\begin{array}{l}\text { BW } \\
\text { BW } \\
\text { BW }\end{array}$ & $\begin{array}{l}\text { Beta, Alpha } \\
\text { Beta, Alpha } \\
\text { Beta, Alpha }\end{array}$ & \multicolumn{2}{|l|}{200 W South East } & Q & ${ }^{90} \mathrm{Sr}, \mathrm{Pu}, \mathrm{U}$, Gamma Scan \\
\hline $200 \mathrm{~W}$ SE & 14 & BW & Beta, Alpha & \multicolumn{2}{|l|}{200 West Area } & $\mathrm{Q}$ & ${ }^{90} \mathrm{Sr}, \mathrm{Pu}, \mathrm{U}, \mathrm{Gamma}$ Scan \\
\hline $\begin{array}{l}300 \text { Water Intake } \\
300 \text { South Gate } \\
300 \text { South West }\end{array}$ & $\begin{array}{l}15 \\
16 \\
17\end{array}$ & $\begin{array}{l}\text { BW } \\
\text { BW } \\
\text { BW }\end{array}$ & $\begin{array}{l}\text { Beta, Alpha } \\
\text { Beta, Alpha } \\
\text { Beta, Alpha }\end{array}$ & \multicolumn{2}{|l|}{300 Area } & $\mathrm{Q}$ & ${ }^{90} \mathrm{Sr}, \mathrm{Pu}, \mathrm{U}$, Gamma Scan \\
\hline 300 Trench & 19 & BW & $\begin{array}{l}\text { Beta,Alpha\} } \\
\text { Beta,Alpha\} }\end{array}$ & $\begin{array}{ll}\text { Q } & \text { U, Gamma } \\
\text { Q } & \text { U, Gamma }\end{array}$ & \multirow[t]{2}{*}{$300 \mathrm{NE}$} & $\mathrm{Q}$ & ${ }^{90} \mathrm{Sr}, \mathrm{Pu}$ \\
\hline $\begin{array}{l}400 \mathrm{E} \\
400 \mathrm{~W} \\
400 \mathrm{~S} \\
400 \mathrm{~N}\end{array}$ & $\begin{array}{l}20 \\
21 \\
22 \\
23\end{array}$ & $\begin{array}{l}\text { BW } \\
\text { BW } \\
\text { BW } \\
\text { BW }\end{array}$ & $\begin{array}{l}\text { Beta , Alpha } \\
\text { Beta , Alpha } \\
\text { Beta , Alpha } \\
\text { Beta , Alpha }\end{array}$ & 400 Area & & $\mathrm{Q}$ & ${ }^{90} \mathrm{Sr}, \mathrm{Pu}, \mathrm{Gamma}$ Scan \\
\hline Wye Barricade & 24 & BW & Beta, Alpha & \multicolumn{2}{|l|}{ Wye Barricade } & $\mathrm{Q}$ & ${ }^{90} \mathrm{Sr}, \mathrm{Pu}, \mathrm{U}, \mathrm{Gamma}$ Scan \\
\hline Ringold Met Tower & 25 & BW & Beta, Alpha & \multicolumn{2}{|l|}{ Ringold Met Tower } & $\mathrm{Q}$ & ${ }^{90} \mathrm{Sr}, \mathrm{Pu}, \mathrm{Gamma}$ Scan \\
\hline W End of Fir Road ${ }^{(b)}$ & 26 & BW & Beta, Alpha & \multicolumn{2}{|c|}{ W End of Fir Road ${ }^{(b)}$} & $\mathrm{Q}$ & ${ }^{90} \mathrm{Sr}, \mathrm{Pu}, \mathrm{U}, \mathrm{Gamma}$ Scan \\
\hline
\end{tabular}




\subsection{AIR - PARTICULATE FILTER (contd)}

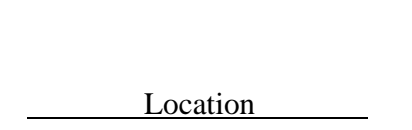

Individual Samples

Location
Number $^{(a)}$

\section{Fre-}

quency

\section{7}

Dogwood Met Tower

Byers Landing

Battelle Complex ${ }^{(b)}$

Horn Rapids Substa

Prosser Barricade

Yakima Barricade

Rattlesnake Springs

Wahluke Slope

S End Vernita Bridge

28

30

31

32

33

34

35 $\underline{\text { Community }}$

Basin City School ${ }^{(\mathrm{c})}$

Leslie Groves-Rchlnd ${ }^{(\mathrm{c})}$

Pasco

Kennewick-Ely Street

Benton City

Edwin Markham School ${ }^{(\mathrm{c})}$

Mattawa

Othello

Distant

Yakima

Toppenish $^{(\mathrm{c})}$

38
39

40
44

45
BW

BW

BW

BW

BW

BW

BW

BW

BW

Beta, Alpha

Beta, Alpha $\}$

Wahluke Slope

BW

Beta, Alpha

Basin City School

BW

Beta, Alpha

Leslie Groves-Rchlnd

BW

Beta

BW

BW

BW

Beta, Alpha

Edwin Markham School

Mattawa

BW

Beta

Othello

BW

Beta, Alpha Yakima

BW

Beta, Alpha

Toppenish
Composited Samples

Fre-

quency
Analyses

Q $\quad{ }^{90} \mathrm{Sr}, \mathrm{Pu}, \mathrm{U}$, Gamma Scan

Q $\quad{ }^{90} \mathrm{Sr}, \mathrm{Pu}, \mathrm{U}$, Gamma Scan

Q Gamma Scan

Q $\quad{ }^{90} \mathrm{Sr}, \mathrm{Pu}, \mathrm{U}, \mathrm{Gamma}$ Scan

Q $\quad{ }^{90} \mathrm{Sr}, \mathrm{Pu}$, Gamma Scan

Q $\quad{ }^{90} \mathrm{Sr}, \mathrm{Pu}, \mathrm{Gamma}$ Scan

Q $\quad{ }^{90} \mathrm{Sr}, \mathrm{Pu}, \mathrm{U}, \mathrm{Gamma}$ Scan

Q $\quad{ }^{90} \mathrm{Sr}, \mathrm{Pu}, \mathrm{U}$, Gamma Scan

Q $\quad{ }^{90} \mathrm{Sr}, \mathrm{Pu}$, Gamma Scan

Q Gamma Scan

Q $\quad{ }^{90} \mathrm{Sr}, \mathrm{Pu}, \mathrm{U}$, Gamma Scan

Q Gamma Scan

Q Gamma Scan

(a) Refer to Figure 1.1, 2002 Air Sampling Locations.

(b) Washington State Department of Health air sampler also at this location.

(c) Community-operated environmental surveillance station. 


\subsection{AIR - TRITIUM AND IODINE}

\begin{tabular}{|c|c|c|c|c|c|}
\hline Location & $\begin{array}{l}\text { Location } \\
\text { Number }^{(a)} \\
\end{array}$ & Frequency $^{(b)}$ & $\underline{\text { Analysis }}$ & Frequency & Analysis $^{(\mathrm{c})}$ \\
\hline \multicolumn{6}{|l|}{$\underline{\text { Onsite }}$} \\
\hline 100 K Area & 1 & & & M & ${ }^{3} \mathrm{H}$ \\
\hline 100 N-1325 Crib & 2 & & & M & ${ }^{3} \mathrm{H}$ \\
\hline $200 \mathrm{ESE}$ & 8 & Q Comp & ${ }^{129} \mathrm{I}$ & M & ${ }^{3} \mathrm{H}$ \\
\hline 200 Tel. Exchange & 12 & & & M & ${ }^{3} \mathrm{H}$ \\
\hline 300 Water Intake & 15 & & & M & ${ }^{3} \mathrm{H}$ \\
\hline 300 South Gate ${ }^{(d)}$ & 16 & & & M & ${ }^{3} \mathrm{H}$ \\
\hline 300 South West & 17 & & & M & ${ }^{3} \mathrm{H}$ \\
\hline 300 Trench & 18 & & & M & ${ }^{3} \mathrm{H}$ \\
\hline $300 \mathrm{NE}$ & 19 & & & M & ${ }^{3} \mathrm{H}$ \\
\hline $400 \mathrm{E}$ & 20 & & & M & ${ }^{3} \mathrm{H}$ \\
\hline \multicolumn{6}{|l|}{$\underline{\text { Perimeter }}$} \\
\hline Ringold Met Tower & 25 & Q Comp & ${ }^{129} \mathrm{I}$ & M & ${ }^{3} \mathrm{H}$ \\
\hline Dogwood Met Tower & 27 & & & M & ${ }^{3} \mathrm{H}$ \\
\hline Byers Landing & 28 & Q Comp & ${ }^{129} \mathrm{I}$ & M & ${ }^{3} \mathrm{H}$ \\
\hline Battelle Complex ${ }^{(\mathrm{e})}$ & 29 & & & M & ${ }^{3} \mathrm{H}$ \\
\hline Prosser Barricade & 31 & & & M & ${ }^{3} \mathrm{H}$ \\
\hline Wahluke Slope & 34 & & & M & ${ }^{3} \mathrm{H}$ \\
\hline \multicolumn{6}{|l|}{ Community $^{(\mathrm{f})}$} \\
\hline Basin City School & 36 & & & M & ${ }^{3} \mathrm{H}$ \\
\hline Leslie Groves-Rchlnd & 37 & & & M & ${ }^{3} \mathrm{H}$ \\
\hline Edwin Markham School & 41 & & & M & ${ }^{3} \mathrm{H}$ \\
\hline \multicolumn{6}{|l|}{$\underline{\text { Distant }}$} \\
\hline Yakima & 44 & Q Comp & ${ }^{129} \mathrm{I}$ & M & ${ }^{3} \mathrm{H}$ \\
\hline Toppenish $^{(\mathrm{f})}$ & 45 & & & M & ${ }^{3} \mathrm{H}$ \\
\hline
\end{tabular}

\footnotetext{
(a) Refer to Figure 1.1, 2002 Air Sampling Locations.

(b) Samples are collected monthly and composited for quarterly analyses.

(c) As HTO.

(d) Two silica gel samples are collected from this location.

(e) Washington State Department of Health air sampler also at this location.

(f) Community-operated environmental surveillance station.
} 


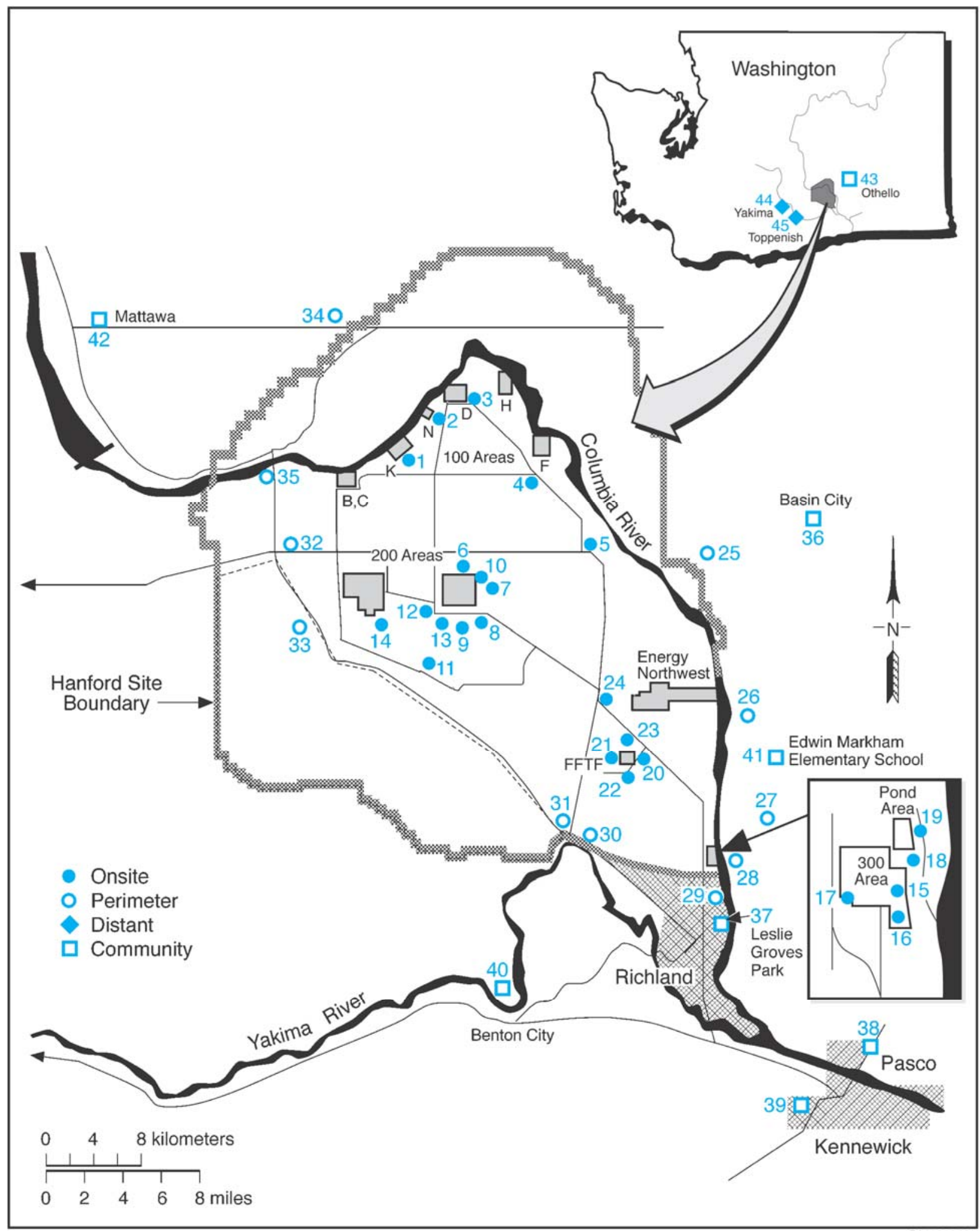

G01120042.

Figure 1.1. 2002 Air Sampling Locations 


\subsection{SURFACE WATER SURVEILLANCE}

\subsection{WATER - COLUMBIA RIVER}

\begin{tabular}{|c|c|c|c|}
\hline Location $^{(\mathrm{a})}$ & Sample Type & Frequency & Analyses \\
\hline Priest Rapids-River & Cumulative & $\begin{array}{l}\text { M Comp. }^{(b)} \\
\text { Q Comp. }^{(b)}\end{array}$ & $\begin{array}{l}\text { Alpha, Beta, Lo }{ }^{3} \mathrm{H},{ }^{90} \mathrm{Sr},{ }^{99} \mathrm{Tc}, \mathrm{U}, \mathrm{DOH}^{(\mathrm{c})} \\
{ }^{129} \mathrm{I}\end{array}$ \\
\hline & Particulate (filter) & $\begin{array}{l}\text { M Comp. } \\
\text { Q Comp. }^{(d)}\end{array}$ & $\begin{array}{l}\text { Gamma Scan } \\
\mathrm{Pu}\end{array}$ \\
\hline & Soluble (resin) & $\begin{array}{l}\text { M Comp. } \\
\text { Q Comp. }^{(d)}\end{array}$ & $\begin{array}{l}\text { Gamma San } \\
\mathrm{Pu}\end{array}$ \\
\hline Rich.Pmphs HRM 46.4 & Cumulative & $\begin{array}{l}\text { M Comp. } \\
\text { Q Comp. }^{(b)}\end{array}$ & $\begin{array}{l}\text { Alpha, Beta, } \mathrm{Lo}{ }^{3} \mathrm{H},{ }^{90} \mathrm{Sr},{ }^{99} \mathrm{Tc}, \mathrm{U} \\
{ }^{129} \mathrm{I}\end{array}$ \\
\hline & Particulate (filter) & $\begin{array}{l}\text { M Comp. } \\
\text { Q Comp. }^{(d)}\end{array}$ & $\begin{array}{l}\text { Gamma Scan } \\
\mathrm{Pu}\end{array}$ \\
\hline & Soluble (resin) & M Comp. $^{(\mathrm{d})}$ & $\begin{array}{l}\text { Gamma Scan } \\
\mathrm{Pu}\end{array}$ \\
\hline & Grab & Q & USGS-NASQAN $^{(\mathrm{e})}$ \\
\hline Rich.Pmphs-1 HRM46.4 & Transect & $\begin{array}{l}\mathrm{Q} \\
\mathrm{A}\end{array}$ & $\begin{array}{l}\text { Lo }{ }^{3} \mathrm{H},{ }^{90} \mathrm{Sr}, \mathrm{U}, \mathrm{ICP}-3 \text {, ICP-3 Filtered, Anions } \\
\text { VOA }\end{array}$ \\
\hline Rich.Pmphs-2 HRM46.4 & Transect & $\mathrm{Q}$ & $\begin{array}{l}\text { Lo }{ }^{3} \mathrm{H},{ }^{90} \mathrm{Sr}, \mathrm{U}, \mathrm{ICP}-3 \text {, ICP-3 Filtered, Anions } \\
\text { VOA }\end{array}$ \\
\hline Rich.Pmphs-3 HRM46.4 & Transect & $\mathrm{Q}$ & $\begin{array}{l}\text { Lo }{ }^{3} \mathrm{H},{ }^{90} \mathrm{Sr}, \mathrm{U}, \mathrm{ICP}-3 \text {, ICP-3 Filtered, Anions } \\
\text { VOA }\end{array}$ \\
\hline Rich.Pmphs-5 HRM46.4 & Transect & $\begin{array}{l}\mathrm{Q} \\
\mathrm{A}\end{array}$ & $\begin{array}{l}\text { Lo }{ }^{3} \mathrm{H},{ }^{90} \mathrm{Sr}, \mathrm{U}, \mathrm{ICP}-3 \text {, ICP-3 Filtered, Anions } \\
\text { VOA }\end{array}$ \\
\hline Rich.Pmphs-7 HRM46.4 & Transect & $\begin{array}{l}\mathrm{Q} \\
\mathrm{A}\end{array}$ & $\begin{array}{l}\text { Lo }{ }^{3} \mathrm{H},{ }^{90} \mathrm{Sr}, \mathrm{U}, \mathrm{ICP}-3 \text {, ICP-3 Filtered, Anions } \\
\text { VOA }\end{array}$ \\
\hline Rich.Pmphs-10 HRM46.4 & Transect & $\mathrm{Q}$ & $\begin{array}{l}\text { Lo }{ }^{3} \mathrm{H},{ }^{90} \mathrm{Sr}, \mathrm{U}, \mathrm{ICP}-3 \text {, ICP-3 Filtered, Anions } \\
\text { VOA }\end{array}$ \\
\hline Rich.Pmphs HRM 43.5 & Transect & $\mathrm{Q}$ & $\begin{array}{l}\text { Lo }{ }^{3} \mathrm{H},{ }^{90} \mathrm{Sr}, \mathrm{U}, \mathrm{ICP}-3 \text {, ICP-3 Filtered, Anions } \\
\text { VOA }\end{array}$ \\
\hline Rich.Pmphs HRM 43.9 & Transect & $\mathrm{Q}$ & $\begin{array}{l}\text { Lo }{ }^{3} \mathrm{H},{ }^{90} \mathrm{Sr}, \mathrm{U}, \mathrm{ICP}-3 \text {, ICP-3 Filtered, Anions } \\
\text { VOA }\end{array}$ \\
\hline Rich.Pmphs HRM 45.0 & Transect & $\begin{array}{l}\mathrm{Q} \\
\mathrm{A}\end{array}$ & $\begin{array}{l}\text { Lo }{ }^{3} \mathrm{H},{ }^{90} \mathrm{Sr}, \mathrm{U}, \mathrm{ICP}-3 \text {, ICP-3 Filtered, Anions } \\
\text { VOA }\end{array}$ \\
\hline Rich.Pmphs HRM 45.8 & Transect & Q & $\begin{array}{l}\text { Lo }{ }^{3} \mathrm{H},{ }^{90} \mathrm{Sr}, \mathrm{U}, \mathrm{ICP}-3 \text {, ICP-3 Filtered, Anions } \\
\text { VOA }\end{array}$ \\
\hline Vernita & Grab & Q & USGS-NASQAN $^{(\mathrm{e})}$ \\
\hline Vernita-1 HRM 0.3 & Transect & $\begin{array}{l}\mathrm{Q} \\
\mathrm{A}\end{array}$ & $\begin{array}{l}\text { Lo }{ }^{3} \mathrm{H},{ }^{90} \mathrm{Sr}, \mathrm{U}, \mathrm{ICP}-3 \text {, ICP-3 Filtered, Anions } \\
\text { VOA }\end{array}$ \\
\hline Vernita-2 HRM 0.3 & Transect & $\begin{array}{l}\mathrm{Q} \\
\mathrm{A}\end{array}$ & $\begin{array}{l}\text { Lo }{ }^{3} \mathrm{H},{ }^{90} \mathrm{Sr}, \mathrm{U}, \mathrm{ICP}-3 \text {, ICP-3 Filtered, Anions } \\
\text { VOA }\end{array}$ \\
\hline
\end{tabular}




\subsection{WATER - COLUMBIA RIVER (contd)}

\begin{tabular}{|c|c|c|c|}
\hline Location $^{(a)}$ & Sample Type & Frequency & Analyses \\
\hline \multirow[t]{2}{*}{ Vernita-3 HRM 0.3} & Transect & Q & Lo ${ }^{3} \mathrm{H},{ }^{90} \mathrm{Sr}, \mathrm{U}, \mathrm{ICP}-3$, ICP-3 Filtered, Anions \\
\hline & & A & VOA \\
\hline \multirow[t]{2}{*}{ Vernita-4 HRM 0.3} & Transect & $\mathrm{Q}$ & Lo ${ }^{3} \mathrm{H},{ }^{90} \mathrm{Sr}, \mathrm{U}, \mathrm{ICP}-3$, ICP-3 Filtered, Anions \\
\hline & & A & VOA \\
\hline 100 N -1 HRM 9.5 & Transect & A & Lo ${ }^{3} \mathrm{H},{ }^{90} \mathrm{Sr}, \mathrm{U}, \mathrm{ICP}-3$, ICP-3 Filtered, Anions \\
\hline 100 N -2 HRM 9.5 & Transect & A & Lo ${ }^{3} \mathrm{H},{ }^{90} \mathrm{Sr}, \mathrm{U}, \mathrm{ICP}-3$, ICP-3 Filtered, Anions \\
\hline 100 N -3 HRM 9.5 & Transect & A & Lo ${ }^{3} \mathrm{H},{ }^{90} \mathrm{Sr}$, U, ICP-3, ICP-3 Filtered, Anions \\
\hline 100 N -5 HRM 9.5 & Transect & A & Lo ${ }^{3} \mathrm{H},{ }^{90} \mathrm{Sr}, \mathrm{U}, \mathrm{ICP}-3$, ICP-3 Filtered, Anions \\
\hline 100 N -7 HRM 9.5 & Transect & A & Lo ${ }^{3} \mathrm{H},{ }^{90} \mathrm{Sr}, \mathrm{U}, \mathrm{ICP}-3$, ICP-3 Filtered, Anions \\
\hline 100 N -10 HRM 9.5 & Transect & A & Lo ${ }^{3} \mathrm{H},{ }^{90} \mathrm{Sr}, \mathrm{U}, \mathrm{ICP}-3$, ICP-3 Filtered, Anions \\
\hline 100 N Shore HRM 8.4 & Transect & A & Lo ${ }^{3} \mathrm{H},{ }^{90} \mathrm{Sr}, \mathrm{U}, \mathrm{ICP}-3$, ICP-3 Filtered, Anions \\
\hline 100 N Shore HRM 8.9 & Transect & A & Lo ${ }^{3} \mathrm{H},{ }^{90} \mathrm{Sr}, \mathrm{U}, \mathrm{ICP}-3$, ICP-3 Filtered, Anions \\
\hline 100 N Shore HRM 9.2 & Transect & A & Lo ${ }^{3} \mathrm{H},{ }^{90} \mathrm{Sr}, \mathrm{U}, \mathrm{ICP}-3$, ICP-3 Filtered, Anions \\
\hline 100 N Shore HRM 9.8 & Transect & A & Lo ${ }^{3} \mathrm{H},{ }^{90} \mathrm{Sr}, \mathrm{U}, \mathrm{ICP}-3$, ICP-3 Filtered, Anions \\
\hline 100 F -1 HRM 19.0 & Transect & A & Lo ${ }^{3} \mathrm{H},{ }^{90} \mathrm{Sr}$, U, ICP-3, ICP-3 Filtered, Anions, DOH ${ }^{(\mathrm{f})}$ \\
\hline 100 F -2 HRM 19.0 & Transect & A & Lo ${ }^{3} \mathrm{H},{ }^{90} \mathrm{Sr}, \mathrm{U}, \mathrm{ICP}-3$, ICP-3 Filtered, Anions, DOH ${ }^{(\mathrm{f})}$ \\
\hline 100 F -3 HRM 19.0 & Transect & A & Lo ${ }^{3} \mathrm{H},{ }^{90} \mathrm{Sr}$, U, ICP-3, ICP-3 Filtered, Anions, DOH ${ }^{(\mathrm{f})}$ \\
\hline 100 F -5 HRM 19.0 & Transect & A & Lo ${ }^{3} \mathrm{H},{ }^{90} \mathrm{Sr}, \mathrm{U}, \mathrm{ICP}-3$, ICP-3 Filtered, Anions, DOH ${ }^{(\mathrm{f})}$ \\
\hline 100 F -7 HRM 19.0 & Transect & A & Lo ${ }^{3} \mathrm{H},{ }^{90} \mathrm{Sr}, \mathrm{U}, \mathrm{ICP}-3$, ICP-3 Filtered, Anions, DOH ${ }^{(\mathrm{f})}$ \\
\hline 100 F -10 HRM 19.0 & Transect & A & Lo ${ }^{3} \mathrm{H},{ }^{90} \mathrm{Sr}, \mathrm{U}, \mathrm{ICP}-3$, ICP-3 Filtered, Anions, DOH ${ }^{(\mathrm{f})}$ \\
\hline 100 F SHORE HRM 18 & Transect & A & $\mathrm{Lo}^{3} \mathrm{H},{ }^{90} \mathrm{Sr}, \mathrm{U}, \mathrm{ICP}-3$, ICP-3 Filtered, Anions, DOH ${ }^{(\mathrm{f})}$ \\
\hline 100 F SHORE HRM 22 & Transect & A & Lo ${ }^{3} \mathrm{H},{ }^{90} \mathrm{Sr}, \mathrm{U}, \mathrm{ICP}-3$, ICP-3 Filtered, Anions, DOH ${ }^{(\mathrm{f})}$ \\
\hline 100 F SHORE HRM 23 & Transect & A & Lo ${ }^{3} \mathrm{H},{ }^{90} \mathrm{Sr}, \mathrm{U}, \mathrm{ICP}-3$, ICP-3 Filtered, Anions, DOH ${ }^{(\mathrm{f})}$ \\
\hline Hanfrd TS-1 HRM 28.7 & Transect & A & Lo ${ }^{3} \mathrm{H},{ }^{90} \mathrm{Sr}, \mathrm{U}, \mathrm{ICP}-3$, ICP-3 Filtered, Anions, DOH ${ }^{(\mathrm{f})}$ \\
\hline Hanfrd TS-2 HRM 28.7 & Transect & A & Lo ${ }^{3} \mathrm{H},{ }^{90} \mathrm{Sr}, \mathrm{U}, \mathrm{ICP}-3$, ICP-3 Filtered, Anions, DOH ${ }^{(\mathrm{f})}$ \\
\hline Hanfrd TS-3 HRM 28.7 & Transect & A & Lo ${ }^{3} \mathrm{H},{ }^{90} \mathrm{Sr}$, U, ICP-3, ICP-3 Filtered, Anions, $\mathrm{DOH}^{(\mathrm{f})}$ \\
\hline Hanfrd TS-5 HRM 28.7 & Transect & A & Lo ${ }^{3} \mathrm{H},{ }^{90} \mathrm{Sr}$, U, ICP-3, ICP-3 Filtered, Anions, $\mathrm{DOH}^{(\mathrm{f})}$ \\
\hline Hanfrd TS-7 HRM 28.7 & Transect & A & Lo ${ }^{3} \mathrm{H},{ }^{90} \mathrm{Sr}$, U, ICP-3, ICP-3 Filtered, Anions, $\mathrm{DOH}^{(\mathrm{f})}$ \\
\hline Hanfrd TS-10 HRM 28.7 & Transect & A & Lo ${ }^{3} \mathrm{H},{ }^{90} \mathrm{Sr}, \mathrm{U}, \mathrm{ICP}-3$, ICP-3 Filtered, Anions, DOH ${ }^{(\mathrm{f})}$ \\
\hline Hanfrd Twnsite HRM26 & Transect & A & Lo ${ }^{3} \mathrm{H},{ }^{90} \mathrm{Sr}, \mathrm{U}, \mathrm{ICP}-3$, ICP-3 Filtered, Anions, $\mathrm{DOH}^{(\mathrm{f})}$ \\
\hline Hanfrd Twnsite HRM27 & Transect & A & Lo ${ }^{3} \mathrm{H},{ }^{90} \mathrm{Sr}, \mathrm{U}, \mathrm{ICP}-3$, ICP-3 Filtered, Anions, DOH ${ }^{(\mathrm{f})}$ \\
\hline Hanfrd Twnsite HRM28 & Transect & A & Lo ${ }^{3} \mathrm{H},{ }^{90} \mathrm{Sr}, \mathrm{U}, \mathrm{ICP}-3$, ICP-3 Filtered, Anions, DOH ${ }^{(\mathrm{f})}$ \\
\hline Hanfrd Twnsite HRM30 & Transect & A & Lo ${ }^{3} \mathrm{H},{ }^{90} \mathrm{Sr}, \mathrm{U}, \mathrm{ICP}-3$, ICP-3 Filtered, Anions, DOH ${ }^{(\mathrm{f})}$ \\
\hline 300 Area-1 HRM 43.1 & Transect & A & Lo ${ }^{3} \mathrm{H},{ }^{90} \mathrm{Sr}, \mathrm{U}, \mathrm{ICP}-3$, ICP-3 Filtered, Anions \\
\hline 300 Area -2 HRM 43.1 & Transect & A & Lo ${ }^{3} \mathrm{H},{ }^{90} \mathrm{Sr}, \mathrm{U}, \mathrm{ICP}-3$, ICP-3 Filtered, Anions \\
\hline 300 Area -3 HRM 43.1 & Transect & A & Lo ${ }^{3} \mathrm{H},{ }^{90} \mathrm{Sr}$, U, ICP-3, ICP-3 Filtered, Anions \\
\hline 300 Area -5 HRM 43.1 & Transect & A & Lo ${ }^{3} \mathrm{H},{ }^{90} \mathrm{Sr}$, U, ICP-3, ICP-3 Filtered, Anions \\
\hline 300 Area -7 HRM 43.1 & Transect & A & Lo ${ }^{3} \mathrm{H},{ }^{90} \mathrm{Sr}$, U, ICP-3, ICP-3 Filtered, Anions \\
\hline 300 Area -10 HRM 43.1 & Transect & A & Lo ${ }^{3} \mathrm{H},{ }^{90} \mathrm{Sr}, \mathrm{U}, \mathrm{ICP}-3$, ICP-3 Filtered, Anions \\
\hline 300 Area Shr HRM41.5 & Transect & A & Lo ${ }^{3} \mathrm{H},{ }^{90} \mathrm{Sr}, \mathrm{U}, \mathrm{ICP}-3$, ICP-3 Filtered, Anions \\
\hline
\end{tabular}




\subsection{WATER - COLUMBIA RIVER (contd)}

\begin{tabular}{|c|c|c|c|}
\hline Location $^{(\mathrm{a})}$ & Sample Type & Frequency & Analyses \\
\hline 300 Area Shr HRM42.1 & Transect & A & Lo ${ }^{3} \mathrm{H},{ }^{90} \mathrm{Sr}, \mathrm{U}, \mathrm{ICP}-3$, ICP-3 Filtered, Anions \\
\hline 300 Area Shr HRM42.5 & Transect & A & Lo ${ }^{3} \mathrm{H},{ }^{90} \mathrm{Sr}, \mathrm{U}, \mathrm{ICP}-3$, ICP-3 Filtered, Anions \\
\hline 300 Area Shr HRM42.9 & Transect & A & Lo ${ }^{3} \mathrm{H},{ }^{90} \mathrm{Sr}, \mathrm{U}, \mathrm{ICP}-3$, ICP-3 Filtered, Anions \\
\hline
\end{tabular}

(a) Refer to Figure 2.1, 2002 Surface Water and Drinking Water Sampling Locations. HRM is referenced to Hanford River mile.

(b) Cumulative sample is collected weekly and composited for analysis.

(c) Cosample provided to the Washington State Department of Health (January and June only).

(d) Sample is collected biweekly and composited for analysis.

(e) Analyses are performed by the United States Geological Survey (USGS) in conjunction with the National Stream Quality Accounting Network (NASQAN) Program, and includes: conductance, $\mathrm{pH}$, temperature, turbidity, dissolved oxygen, hardness, Ca, Mg, alkalinity, carbonates, sulfate, $\mathrm{CI}, \mathrm{F}$, solids, $\mathrm{NH}_{4}-\mathrm{N}, \mathrm{NO}_{3}+\mathrm{NO}_{2}$, N-Kjeldahl, $\mathrm{P}, \mathrm{Cr}$, Fe, dissolved organic carbon.

(f) Cosample provided to the Washington State Department of Health (September).

\subsection{RIVERBANK SPRINGS}

\begin{tabular}{|c|c|c|c|}
\hline Location $^{(a)}$ & $\begin{array}{l}\text { Sample } \\
\text { Type }\end{array}$ & Frequency & Analyses \\
\hline 100-B Spring 38-3 & Grab & A & Alpha, Beta, ${ }^{3} \mathrm{H},{ }^{90} \mathrm{Sr},{ }^{99} \mathrm{Tc}$, Gamma Scan, ICP-3, ICP-3 Filtered, Anions, VOA \\
\hline 100-B Spring 39-2 & Grab & A & Alpha, Beta, ${ }^{3} \mathrm{H},{ }^{90} \mathrm{Sr},{ }^{99} \mathrm{Tc}$, Gamma Scan, ICP-3, ICP-3 Filtered, Anions, VOA, DOH ${ }^{(\mathrm{b})}$ \\
\hline 100-K Spring 63-1 & Grab & A & Alpha, Beta, ${ }^{3} \mathrm{H},{ }^{90} \mathrm{Sr}$, Gamma Scan, ICP-3, ICP-3 Filtered, Anions, VOA, DOH ${ }^{(\mathrm{b})}$ \\
\hline 100-K Spring 77-1 & Grab & A & Alpha, Beta, ${ }^{3} \mathrm{H},{ }^{90} \mathrm{Sr}$, Gamma Scan, ICP-3, ICP-3 Filtered, Anions, VOA, DOH ${ }^{(b)}$ \\
\hline 100-N Spring 8-13 & Grab & A & Alpha, Beta, ${ }^{3} \mathrm{H},{ }^{90} \mathrm{Sr}$, Gamma Scan, ICP-3, ICP-3 Filtered, Anions \\
\hline $\begin{array}{l}\text { 100-N Spring } \\
\quad \text { Near 199N-46 }\end{array}$ & Grab & A & Alpha, Beta, ${ }^{3} \mathrm{H},{ }^{90} \mathrm{Sr}$, Gamma Scan, ICP-3, ICP-3 Filtered, Anions, DOH ${ }^{(b)}$ \\
\hline 100-D Spring 110-1 & Grab & A & Alpha, Beta, ${ }^{3} \mathrm{H},{ }^{90} \mathrm{Sr}$, Gamma Scan, ICP-3, ICP-3 Filtered, Anions \\
\hline 100-D Spring 102-1 & Grab & A & Alpha, Beta, ${ }^{3} \mathrm{H},{ }^{90} \mathrm{Sr}$, Gamma Scan, ICP-3, ICP-3 Filtered, Anions \\
\hline 100-H Spring 152-2 & Grab & A & Alpha, Beta, ${ }^{3} \mathrm{H},{ }^{90} \mathrm{Sr},{ }^{99} \mathrm{Tc}, \mathrm{U}$, Gamma Scan, ICP-3, ICP-3 Filtered, Anions \\
\hline 100-H Spring $145-1$ & Grab & A & Alpha, Beta, ${ }^{3} \mathrm{H},{ }^{90} \mathrm{Sr},{ }^{99} \mathrm{Tc}, \mathrm{U}$, Gamma Scan, ICP-3, ICP-3 Filtered, Anions, DOH ${ }^{(\mathrm{b})}$ \\
\hline 100-F Spring 207-1 & Grab & A & Alpha, Beta, ${ }^{3} \mathrm{H},{ }^{90} \mathrm{Sr}, \mathrm{U}$, Gamma Scan, ICP-3, ICP-3 Filtered, Anions, VOA, DOH ${ }^{(\mathrm{b})}$ \\
\hline Hanford Spring 28-2 & Grab & A & Alpha, Beta, ${ }^{3} \mathrm{H},{ }^{99} \mathrm{Tc}, \mathrm{U},{ }^{129} \mathrm{I}, \mathrm{Gamma}$ Scan, ICP-3, ICP-3 Filtered, Anions, DOH ${ }^{(\mathrm{b})}$ \\
\hline Hanford Spr UR 28-2 & Grab & A & Alpha, Beta, ${ }^{3} \mathrm{H},{ }^{99} \mathrm{Tc}, \mathrm{U},{ }^{129}$ I, Gamma Scan, ICP-3, ICP-3 Filtered, Anions \\
\hline Hanford Spr DR 28-2 & Grab & A & Alpha, Beta, ${ }^{3} \mathrm{H},{ }^{99} \mathrm{Tc}, \mathrm{U},{ }^{129} \mathrm{I}$, Gamma Scan, ICP-3, ICP-3 Filtered, Anions \\
\hline 300 Area Spring 42-2 & Grab & A & Alpha, Beta, ${ }^{3} \mathrm{H},{ }^{90} \mathrm{Sr}, \mathrm{U},{ }^{129} \mathrm{I}$, Gamma Scan, ICP-3, ICP-3 Filtered, Anions, VOA \\
\hline 300 Area Spr DR 42-2 & Grab & A & Alpha, Beta, ${ }^{3} \mathrm{H},{ }^{90} \mathrm{Sr}, \mathrm{U},{ }^{129} \mathrm{I}$, Gamma Scan, ICP-3, ICP-3 Filtered, Anions, VOA \\
\hline
\end{tabular}

(a) Refer to Figure 2.1, 2002 Surface Water and Drinking Water Sampling Locations.

(b) Cosample provided to the Washington State Department of Health. 


\subsection{ONSITE PONDS}

\begin{tabular}{|c|c|c|c|}
\hline Location $^{(a)}$ & Sample Type & $\underline{\text { Frequency }}$ & Analyses \\
\hline West Lake & Grab & Q & Alpha, Beta, ${ }^{3} \mathrm{H},{ }^{99} \mathrm{Tc}, \mathrm{U}$, Gamma Scan \\
\hline FFTF Pond & Grab & $\mathrm{Q}$ & Alpha, Beta, ${ }^{3} \mathrm{H}$, Gamma Scan \\
\hline
\end{tabular}

(a) Refer to Figure 2.1, 2002 Surface Water and Drinking Water Sampling Locations.

\subsection{OFFSITE IRRIGATION WATER}

\begin{tabular}{|c|c|c|c|}
\hline Location $^{(a)}$ & Sample Type & Frequency & Analyses \\
\hline Riverview Canal & Grab & 3 (May-Sept) & Alpha, Beta, Lo ${ }^{3} \mathrm{H},{ }^{90} \mathrm{Sr}$, U, Gamma Scan, DOH ${ }^{(\mathrm{b})}$ \\
\hline Horn Rapids Area & Grab & 3 (May-Sept) & Alpha, Beta, Lo ${ }^{3} \mathrm{H},{ }^{90} \mathrm{Sr}, \mathrm{U}$, Gamma Scan, $\mathrm{DOH}^{(\mathrm{b})}$ \\
\hline
\end{tabular}

(a) Refer to Figure 2.1, 2002 Surface Water and Drinking Water Sampling Locations.

(b) One cosample provided to the Washington State Department of Health.

\subsection{ONSITE DRINKING WATER}

\begin{tabular}{|c|c|c|c|}
\hline Location $^{(a)}$ & Sample Type & Frequency & Analyses \\
\hline 100 N Area & Grab & Q & Alpha, Beta, ${ }^{3} \mathrm{H},{ }^{90} \mathrm{Sr}, \mathrm{DOH}^{(\mathrm{b})}$ \\
\hline 200 W Area & Grab & Q & Alpha, Beta, ${ }^{3} \mathrm{H},{ }^{90} \mathrm{Sr}$ \\
\hline $100 \mathrm{~K}$ Area & Grab & Q & Alpha, Beta, ${ }^{3} \mathrm{H},{ }^{90} \mathrm{Sr}$ \\
\hline 400 Area & Grab & Q & Alpha, Beta, ${ }^{3} \mathrm{H},{ }^{90} \mathrm{Sr}, \mathrm{DOH}^{(\mathrm{b})}$ \\
\hline
\end{tabular}

(a) Refer to Figure 2.1, 2002 Surface Water and Drinking Water Sampling Locations.

(b) During 2nd quarter, cosample provided to the Washington State Department of Health. 


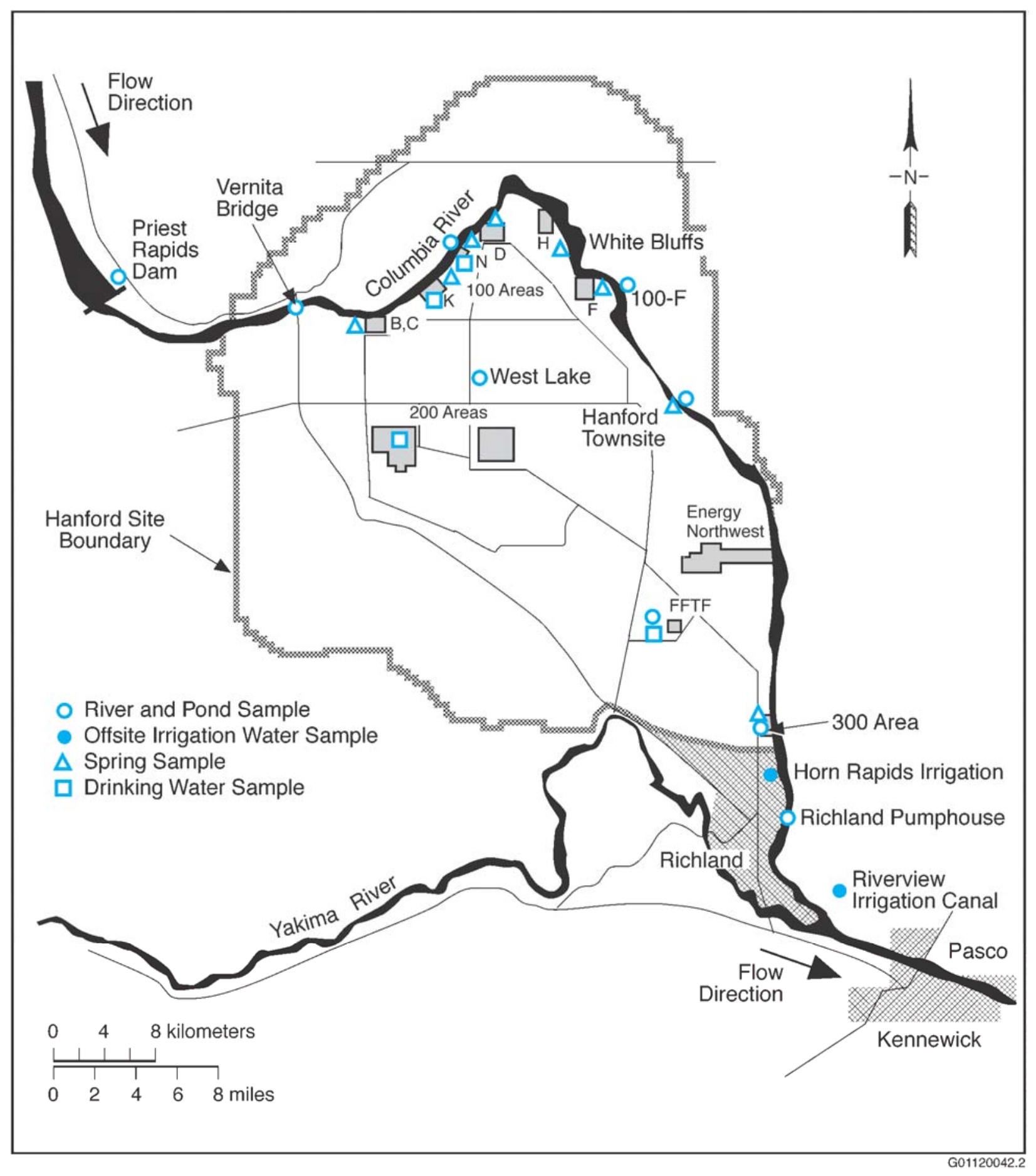

Figure 2.1. 2002 Surface Water and Drinking Water Sampling Locations 


\subsection{BIOTA}

\subsection{FOODSTUFFS AND FARM PRODUCTS}

\subsubsection{Whole Milk}

\begin{tabular}{|c|c|c|}
\hline Location $^{(a)}$ & Frequency & Analyses \\
\hline \multirow[t]{2}{*}{ East Wahluke Area ${ }^{(b)}$} & $\mathrm{Q}$ & \multirow{2}{*}{$\begin{array}{l}\mathrm{Lo}^{3} \mathrm{H},{ }^{90} \mathrm{Sr} \text {, Gamma Scan } \\
{ }_{\mathrm{I}}\end{array}$} \\
\hline & SA & \\
\hline \multirow[t]{2}{*}{ Sagemoor Composite $^{(b)}$} & Q & \multirow{2}{*}{$\begin{array}{l}\text { Lo }{ }^{3} \mathrm{H},{ }^{90} \mathrm{Sr} \text {, Gamma Scan } \\
{ }^{129} \mathrm{I}\end{array}$} \\
\hline & SA & \\
\hline \multirow[t]{2}{*}{ Sunnyside Area } & $\mathrm{Q}$ & \multirow{2}{*}{$\begin{array}{l}\text { Lo }{ }^{3} \mathrm{H},{ }^{90} \mathrm{Sr} \text {, Gamma Scan } \\
{ }^{129} \mathrm{I}\end{array}$} \\
\hline & SA & \\
\hline
\end{tabular}

(a) Refer to Figure 3.1, 2002 Food and Farm Product Sampling Locations.

(b) Sample composited from multiple dairies in each area.

\subsubsection{Leafy Vegetables}

Location $^{(\mathrm{a})(\mathrm{b})}$

Riverview Area

Sunnyside Area

East Wahluke Area

Sagemoor Area

\section{Frequency $^{(\mathrm{c})}$}

A

A

BE (2002)

BE (2003)
Analyses

${ }^{90}$ Sr, Gamma Scan, FDA ${ }^{(\mathrm{d})}, \mathrm{DOH}^{(\mathrm{e})}$
${ }^{90} \mathrm{Sr}$, Gamma Scan, FDA
${ }^{(\mathrm{d})}$
${ }^{90} \mathrm{Sr}$, Gamma Scan, $\mathrm{DOH}^{(\mathrm{e})}$
${ }^{90} \mathrm{Sr}$, Gamma Scan, $\mathrm{DOH}^{(\mathrm{e})}$

(a) Refer to Figure 3.1, 2002 Food and Farm Product Sampling Locations.

(b) Two samples collected within each area, one sample analyzed and one archived.

(c) Samples are collected in 2002 according to their specified frequency unless otherwise noted.

(d) Cosamples sent to U.S. Food and Drug Administration.

(e) Cosample provided to the Washington State Department of Health.

\subsubsection{Vegetables}

Location $^{(\mathrm{a})(\mathrm{b})}$

Riverview Area

Sunnyside Area

East Wahluke Area

Harrah/Wapato Area ${ }^{(\mathrm{f})}$

Horn Rapids Area

Sagemoor Area $\underline{\text { Sample Type }}$

Potatoes
Tomatoes
Potatoes
Potatoes
Tomatoes
Potatoes
Potatoes

Frequency $^{(\mathrm{c})}$

A

A

A

A

A

TE (2002)

TE (2003)
Analyses

${ }^{90}$ Sr, Gamma Scan, DOH ${ }^{(\mathrm{d})}$

${ }^{90} \mathrm{Sr},{ }^{3} \mathrm{H}$, Gamma Scan

${ }^{90} \mathrm{Sr}$, Gamma Scan, FDA ${ }^{(\mathrm{e})}$

${ }^{90} \mathrm{Sr}$, Gamma Scan, DOH ${ }^{(\mathrm{d})}$

${ }^{90} \mathrm{Sr},{ }^{3} \mathrm{H}$, Gamma Scan, DOH

${ }^{90} \mathrm{Sr}$, Gamma Scan, $\mathrm{DOH}^{(\mathrm{d})}, \mathrm{FDA}^{(\mathrm{e})}$

${ }^{90} \mathrm{Sr}$, Gamma Scan, $\mathrm{DOH}^{(\mathrm{d})}, \mathrm{FDA}^{(\mathrm{e})}$

(a) Refer to Figure 3.1, 2002 Food and Farm Product Sampling Locations.

(b) Two samples collected within each area, one sample analyzed and one archived.

(c) Samples are collected in 2002 according to their specified frequency unless otherwise noted.

(d) Cosample provided to the Washington State Department of Health.

(e) Cosamples sent to U.S. Food and Drug Administration.

(f) Samples provided to PNNL by Washington State Department of Health. 


\subsubsection{Fruit}

\begin{tabular}{|c|c|c|c|c|}
\hline Location $^{(\mathrm{a})(\mathrm{b})}$ & Sample Type & Frequency $^{(\mathrm{c})}$ & $\begin{array}{c}\text { Collection } \\
\text { Period } \\
\end{array}$ & Analyses \\
\hline \multirow[t]{3}{*}{ Sagemoor Area } & Cherries & TE (2002) & June & ${ }^{90} \mathrm{Sr}$, Gamma Scan, $\mathrm{DOH}^{(\mathrm{d})}, \mathrm{FDA}^{(\mathrm{e})}$ \\
\hline & Apples & TE (2003) & September & ${ }^{90} \mathrm{Sr}$, Gamma Scan, $\mathrm{DOH}^{(\mathrm{d})}, \mathrm{FDA}^{(\mathrm{e})}$ \\
\hline & Concord Grapes $^{(\mathrm{f})}$ & TE (2004) & September & ${ }^{90} \mathrm{Sr}$, Gamma Scan, $\mathrm{DOH}^{(\mathrm{d})}$ \\
\hline \multirow[t]{3}{*}{ Sunnyside Area } & Cherries & TE (2002) & June & ${ }^{90} \mathrm{Sr}$, Gamma Scan, $\mathrm{DOH}^{(\mathrm{d})}$ \\
\hline & Apples & TE (2003) & September & ${ }^{90}$ Sr, Gamma Scan \\
\hline & Concord Grapes $^{(\mathrm{f})}$ & TE (2004) & September & ${ }^{90} \mathrm{Sr}$, Gamma Scan, $\mathrm{DOH}^{(\mathrm{d})}$ \\
\hline \multirow[t]{3}{*}{ Riverview Area } & Cherries & TE (2002) & June & ${ }^{90} \mathrm{Sr}$, Gamma Scan, DOH ${ }^{(\mathrm{d})}$ \\
\hline & Apples & TE (2003) & September & ${ }^{90} \mathrm{Sr}$, Gamma Scan, $\mathrm{DOH}^{(\mathrm{d})}, \mathrm{FDA}^{(\mathrm{e})}$ \\
\hline & Concord Grapes $^{(\mathrm{f})}$ & TE (2004) & September & ${ }^{90} \mathrm{Sr}$, Gamma Scan, $\mathrm{DOH}^{(\mathrm{d})}, \mathrm{FDA}^{(\mathrm{e})}$ \\
\hline Ringold Area & Cherries & TE (2002) & June & ${ }^{90}$ Sr, Gamma Scan, DOH ${ }^{(\mathrm{d})}$ \\
\hline East Wahluke Area & Cherries & TE (2002) & June & ${ }^{90} \mathrm{Sr}$, Gamma Scan \\
\hline Mattawa Area & Apples & TE (2003) & September & ${ }^{90}$ Sr, Gamma Scan, DOH ${ }^{(\mathrm{d})}$ \\
\hline Cold Creek Area & Concord Grapes $^{(\mathrm{f})}$ & TE (2004) & September & ${ }^{90} \mathrm{Sr}$, Gamma Scan, DOH ${ }^{(\mathrm{d})}$ \\
\hline \multicolumn{5}{|c|}{ (a) Refer to Figure 3.1, 2002 Food and Farm Product Sampling Locations. } \\
\hline \multicolumn{5}{|c|}{ (b) Two samples collected within each area, one sample analyzed and one archived. } \\
\hline \multicolumn{5}{|c|}{ (c) Samples are collected in 2002 according to their specified frequency unless otherwise noted. } \\
\hline \multicolumn{5}{|c|}{ (d) Cosample provided to the Washington State Department of Health. } \\
\hline \multicolumn{5}{|c|}{ (e) Cosamples sent to U.S. Food and Drug Administration. } \\
\hline (f) Concord grapes & red; table grapes ac & & & \\
\hline
\end{tabular}

\subsubsection{Wine}

\begin{tabular}{|c|c|c|c|c|}
\hline Location $^{(a)(b)}$ & $\underline{\text { Sample Type }}$ & Frequency & $\begin{array}{l}\text { Collection } \\
\text { Period } \\
\end{array}$ & Analyses \\
\hline \multirow[t]{2}{*}{ Columbia Basin } & White & A & December & $\mathrm{Lo}^{3} \mathrm{H}$, Gamma Scan, $\mathrm{DOH}^{(\mathrm{c})}$ \\
\hline & Red & A & December & Lo $^{3} \mathrm{H}$, Gamma Scan, DOH ${ }^{(\mathrm{c})}$ \\
\hline \multirow[t]{2}{*}{ Yakima Valley } & White & A & December & Lo ${ }^{3} \mathrm{H}$, Gamma Scan, DOH ${ }^{(\mathrm{c})}$ \\
\hline & Red & A & December & $\mathrm{Lo}^{3} \mathrm{H}$, Gamma Scan, $\mathrm{DOH}^{(\mathrm{c})}$ \\
\hline
\end{tabular}

(a) Refer to Figure 3.1, 2002 Food and Farm Product Sampling Locations.

(b) Two samples of each type collected within each area.

(c) Cosample provided to the Washington State Department of Health. 


\subsubsection{Alfalfa}

\begin{tabular}{|c|c|c|c|c|}
\hline Location $^{(\mathrm{a})}$ & $\underline{\text { Sample Type }}$ & Frequency & $\begin{array}{c}\text { Collection } \\
\text { Period } \\
\end{array}$ & Analyses \\
\hline Sagemoor Area & Alfalfa & BE (2003) & May & ${ }^{90} \mathrm{Sr}$, Gamma Scan \\
\hline Riverview Area & Alfalfa & BE (2003) & May & ${ }^{90} \mathrm{Sr}$, Gamma Scan, FDA ${ }^{(\mathrm{b})}, \mathrm{DOH}^{(\mathrm{c})}$ \\
\hline Sunnyside Area & Alfalfa & BE (2003) & May & ${ }^{90} \mathrm{Sr}$, Gamma Scan, FDA ${ }^{(\mathrm{b})}$ \\
\hline Horn Rapids Area & Alfalfa & BE (2003) & May & ${ }^{90} \mathrm{Sr}$, Gamma Scan, $\mathrm{DOH}^{(\mathrm{c})}$ \\
\hline
\end{tabular}

(a) Two samples collected within each area, one sample analyzed and one archived.

(b) Cosamples sent to U.S. Food and Drug Administration.

(c) Cosample provided to the Washington State Department of Health. 


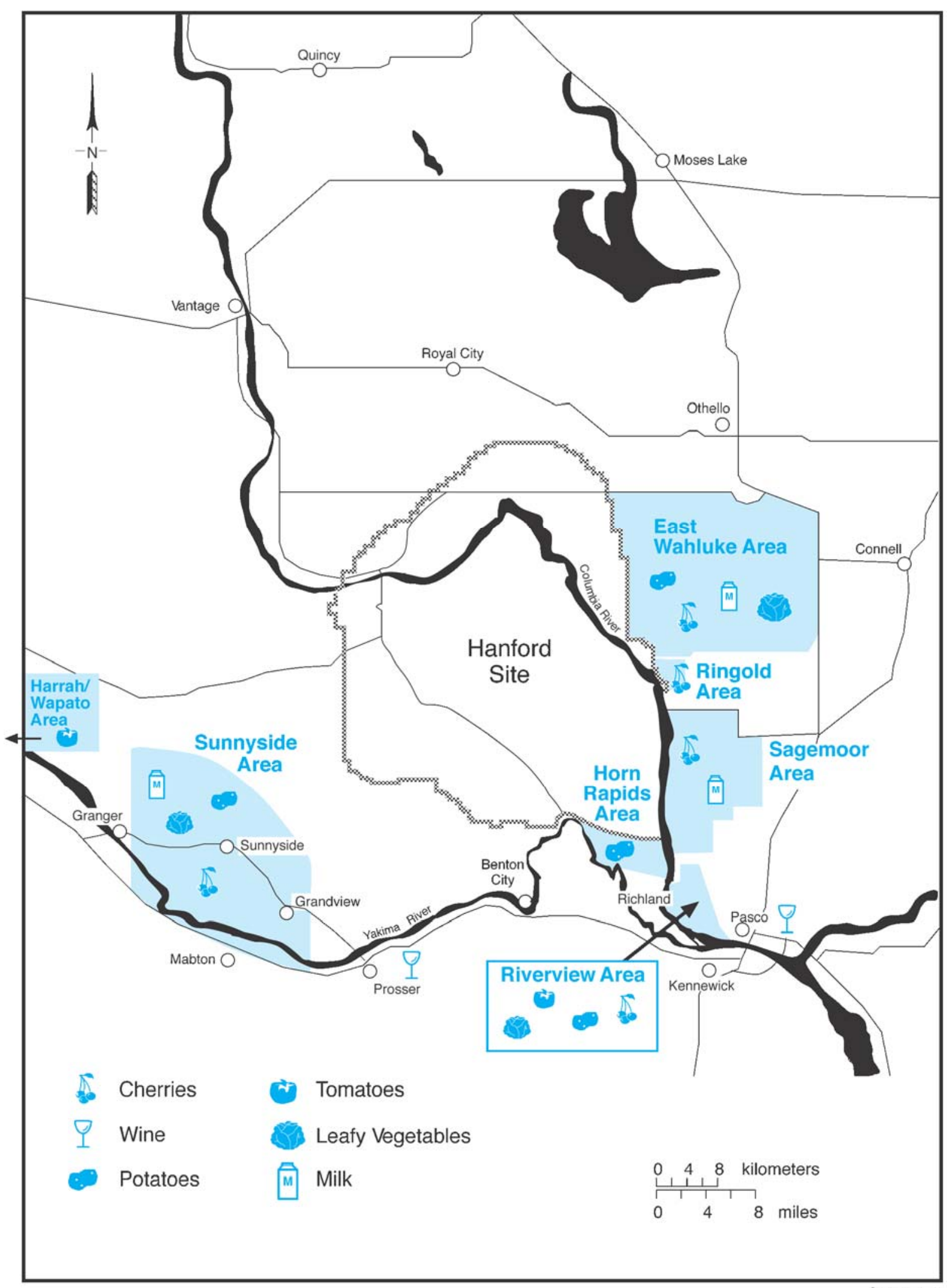

Figure 3.1. 2002 Food and Farm Product Sampling Locations 


\subsection{WILDLIFE}

\subsubsection{Aquatic Biota}

\begin{tabular}{|c|c|c|c|c|c|}
\hline Location $^{(a)}$ & $\begin{array}{l}\text { Species/ } \\
\text { Sample }\end{array}$ & $\begin{array}{c}\text { Number } \\
\text { of Samples } \\
\end{array}$ & Frequency $^{(\mathrm{b})}$ & $\begin{array}{l}\text { Collection } \\
\text { Period } \\
\end{array}$ & Analyses \\
\hline $100 \mathrm{~N}$ Area to & Carp & & & & \multirow{3}{*}{$\begin{array}{l}\text { Gamma Scan, } \mathrm{DOH}^{(\mathrm{d})} \\
{ }^{90} \mathrm{Sr}, \mathrm{DOH}^{(\mathrm{d})}\end{array}$} \\
\hline \multirow{5}{*}{100 D Area ${ }^{(c)}$} & Fillet & 5 & BE (2002) & June & \\
\hline & Carcass & 5 & BE (2002) & June & \\
\hline & Whitefish & & & & \\
\hline & Fillet & 5 & BE (2003) & November & Gamma Scan, DOH ${ }^{(\mathrm{d})}$ \\
\hline & Carcass & 5 & BE (2003) & November & ${ }^{90} \mathrm{Sr}, \mathrm{DOH}^{(\mathrm{d})}$ \\
\hline \multirow[t]{3}{*}{100 F Slough } & Bass & & & & \multirow{3}{*}{$\begin{array}{l}\text { Gamma Scan, } \mathrm{DOH}^{(\mathrm{d})} \\
{ }^{90} \mathrm{Sr}, \mathrm{DOH}^{(\mathrm{d})}\end{array}$} \\
\hline & Fillet & 5 & TE (2002) & May-June & \\
\hline & Carcass & 5 & TE (2002) & May-June & \\
\hline \multirow[t]{3}{*}{ Hanford Slough } & Bass & & & & \multirow{3}{*}{$\begin{array}{l}\text { Gamma Scan, } \mathrm{DOH}^{(\mathrm{d})} \\
{ }^{90} \mathrm{Sr}, \mathrm{DOH}^{(\mathrm{d})}\end{array}$} \\
\hline & Fillet & 5 & TE (2002) & May-June & \\
\hline & Carcass & 5 & TE (2002) & May-June & \\
\hline \multirow[t]{6}{*}{300 Area $^{(c)}$} & Carp & & & & \\
\hline & Fillet & 5 & BE (2002) & June & \multirow{2}{*}{$\begin{array}{l}\text { Gamma Scan, U, DOH } \\
{ }^{(\mathrm{d})} \\
{ }^{90} \mathrm{Sr}, \mathrm{DOH}^{(\mathrm{d})}\end{array}$} \\
\hline & Carcass & 5 & BE (2002) & June & \\
\hline & Bass & & & & \\
\hline & Fillet & 5 & TE (2002) & May-June & Gamma Scan, U \\
\hline & Carcass & 5 & TE (2002) & May-June & ${ }^{90} \mathrm{Sr}$ \\
\hline \multirow[t]{3}{*}{ Desert Aire } & Bass & & & & \\
\hline & Fillet & 5 & TE (2002) & June & \multirow{2}{*}{$\begin{array}{l}\text { Gamma Scan, U, DOH } \\
{ }^{(\mathrm{d})} \\
{ }^{90} \mathrm{Sr}, \mathrm{DOH}^{(\mathrm{d})}\end{array}$} \\
\hline & Carcass & 5 & TE (2002) & June & \\
\hline \multirow[t]{3}{*}{ Vantage } & Carp & & & & \multirow{3}{*}{$\begin{array}{l}\text { Gamma Scan, U } \\
{ }^{90} \mathrm{Sr}\end{array}$} \\
\hline & Fillet & 5 & BE (2002) & June & \\
\hline & Carcass & 5 & BE (2002) & June & \\
\hline \multirow[t]{3}{*}{ Background } & Whitefish & & & & \\
\hline & Fillet & 5 & TE (2005) & Jan \& Dec & \multirow{2}{*}{$\begin{array}{l}\text { Gamma Scan } \\
{ }^{90} \mathrm{Sr}\end{array}$} \\
\hline & Carcass & 5 & TE (2005) & Jan \& Dec & \\
\hline
\end{tabular}

\footnotetext{
(a) Refer to Figure 3.2, 2002 Wildlife Sampling Locations.

(b) Samples are collected in 2002 according to their specified frequency unless otherwise noted.

(c) If available, PNNL will collect one Squawfish sample and provide to the Washington State Department of Health.

(d) One cosample provided to the Washington State Department of Health.
} 


\subsubsection{Geese}

\begin{tabular}{|c|c|c|c|c|c|}
\hline Location & $\underline{\text { Species/Sample }}$ & $\begin{array}{c}\text { Number } \\
\text { of Samples }\end{array}$ & Frequency & $\begin{array}{l}\text { Collection } \\
\text { Period } \\
\end{array}$ & Analyses \\
\hline \multirow[t]{3}{*}{100 Areas } & Canada Goose & & & & \\
\hline & Muscle & 5 & BE (2003) & August & \multirow{2}{*}{$\begin{array}{l}\text { Gamma Scan, } \mathrm{DOH}^{(\mathrm{a}} \\
{ }^{90} \mathrm{Sr}, \mathrm{DOH}^{(\mathrm{a})}\end{array}$} \\
\hline & Bone & 5 & BE (2003) & August & \\
\hline \multirow[t]{3}{*}{ Hanford Townsite } & Canada Goose & & & & \\
\hline & Muscle & 5 & BE (2003) & August & \multirow{2}{*}{$\begin{array}{l}\text { Gamma Scan } \\
{ }^{90} \mathrm{Sr}\end{array}$} \\
\hline & Bone & 5 & BE (2003) & August & \\
\hline \multirow[t]{3}{*}{ Vantage } & Canada Goose & & & & \\
\hline & Muscle & 5 & BE (2003) & August & \multirow{2}{*}{$\begin{array}{l}\text { Gamma Scan } \\
{ }^{90} \mathrm{Sr}\end{array}$} \\
\hline & Bone & 5 & BE (2003) & August & \\
\hline
\end{tabular}

(a) One cosample provided to the Washington State Department of Health.

\subsubsection{Game Birds}

\begin{tabular}{|c|c|c|c|c|c|}
\hline Location $^{\text {(a) }}$ & Species/Sample ${ }^{(a)}$ & $\begin{array}{c}\text { Number } \\
\text { of Samples }\end{array}$ & Frequency $^{(\mathrm{c})}$ & $\begin{array}{c}\text { Collection } \\
\text { Period }\end{array}$ & Analyses \\
\hline $\begin{array}{l}100 \mathrm{D} \text { Area to } \\
100 \mathrm{H} \text { Area }\end{array}$ & $\begin{array}{c}\text { Pheasant } \\
\text { Muscle } \\
\text { Bone }\end{array}$ & $\begin{array}{l}4 \\
4\end{array}$ & $\begin{array}{l}\text { BE (2002) } \\
\text { BE (2002) }\end{array}$ & $\begin{array}{l}\text { September } \\
\text { September }\end{array}$ & $\begin{array}{l}\text { Gamma Scan, } \mathrm{DOH}^{(\mathrm{d})} \\
{ }^{90} \mathrm{Sr}, \mathrm{DOH}^{(\mathrm{d})}\end{array}$ \\
\hline $\begin{array}{l}100 \mathrm{H} \text { Area to } \\
100 \text { F Area }\end{array}$ & $\begin{array}{c}\text { Pheasant } \\
\text { Muscle } \\
\text { Bone }\end{array}$ & $\begin{array}{l}6 \\
6\end{array}$ & $\begin{array}{l}\text { BE (2002) } \\
\text { BE (2002) }\end{array}$ & $\begin{array}{l}\text { September } \\
\text { September }\end{array}$ & $\begin{array}{l}\text { Gamma Scan, } \mathrm{DOH}^{(\mathrm{d})} \\
{ }^{90} \mathrm{Sr}, \mathrm{DOH}^{(\mathrm{d})}\end{array}$ \\
\hline Background & $\begin{array}{c}\text { Pheasant } \\
\text { Muscle } \\
\text { Bone }\end{array}$ & $\begin{array}{l}5 \\
5\end{array}$ & $\begin{array}{l}\mathrm{BE}(2002) \\
\mathrm{BE}(2002)\end{array}$ & $\begin{array}{l}\text { September } \\
\text { September }\end{array}$ & $\begin{array}{l}\text { Gamma Scan } \\
{ }^{90} \mathrm{Sr}\end{array}$ \\
\hline
\end{tabular}

(a) Refer to Figure 3.2, 2002 Wildlife Sampling Locations.

(b) Pheasant preferred; chukar or quail acceptable if pheasant is unavailable.

(c) Samples are collected in 2002 according to their specified frequency unless otherwise noted.

(d) One cosample provided to the Washington State Department of Health. 


\subsubsection{Rabbits}

\begin{tabular}{|c|c|c|c|c|c|}
\hline Location & Species/Sample & $\begin{array}{c}\text { Number } \\
\text { of Samples }\end{array}$ & Frequency & $\begin{array}{l}\text { Collection } \\
\text { Period } \\
\end{array}$ & Analyses \\
\hline \multirow[t]{3}{*}{100 N Area } & Cottontail & & & & \multirow{3}{*}{$\begin{array}{l}\text { Gamma Scan, } \mathrm{DOH}^{(\mathrm{a})} \\
{ }^{90} \mathrm{Sr}, \mathrm{DOH}^{(\mathrm{a})}\end{array}$} \\
\hline & Muscle & 4 & BE (2003) & April & \\
\hline & Bone & 4 & BE (2003) & April & \\
\hline \multirow[t]{3}{*}{200 E Area } & Cottontail & & & & \multirow{3}{*}{$\begin{array}{l}\text { Gamma Scan } \\
{ }^{90} \mathrm{Sr}\end{array}$} \\
\hline & Muscle & 4 & BE (2003) & April & \\
\hline & Bone & 4 & BE (2003) & April & \\
\hline \multirow[t]{3}{*}{200 West } & Cottontail & & & & \multirow{3}{*}{$\begin{array}{l}\text { Gamma Scan } \\
{ }^{90} \mathrm{Sr}\end{array}$} \\
\hline & Muscle & 4 & BE (2003) & April & \\
\hline & Bone & 4 & BE (2003) & April & \\
\hline \multirow[t]{3}{*}{ Background } & Cottontail & & & & \multirow{3}{*}{$\begin{array}{l}\text { Gamma Scan } \\
{ }^{90} \mathrm{Sr}\end{array}$} \\
\hline & Muscle & 5 & TE (2005) & April & \\
\hline & Bone & 5 & TE (2005) & April & \\
\hline
\end{tabular}

(a) One cosample provided to the Washington State Department of Health.

\subsubsection{Deer/Elk}

\begin{tabular}{|c|c|c|c|c|c|}
\hline Location $^{\text {(a) }}$ & $\begin{array}{l}\text { Species/ } \\
\text { Sample }\end{array}$ & $\begin{array}{c}\text { Number } \\
\text { of Samples }\end{array}$ & Frequency $^{(\mathrm{b})}$ & $\begin{array}{l}\text { Collection } \\
\text { Period }\end{array}$ & Analyses \\
\hline \multirow[t]{3}{*}{100 N Area } & Mule Deer & & & & \\
\hline & Muscle & 2 & BE (2002) & December & \multirow{2}{*}{$\begin{array}{l}\text { Gamma Scan, } \mathrm{DOH}^{(\mathrm{c})} \\
{ }^{90} \mathrm{Sr}, \mathrm{DOH}^{(\mathrm{c})}\end{array}$} \\
\hline & Bone & 2 & BE (2002) & December & \\
\hline \multirow[t]{4}{*}{200 Areas } & Mule Deer & & & & \multirow{4}{*}{$\begin{array}{l}\text { Gamma Scan, } \mathrm{DOH}^{(\mathrm{c})} \\
{ }^{90} \mathrm{Sr}, \mathrm{DOH}^{(\mathrm{c})} \\
\mathrm{Pu}\end{array}$} \\
\hline & Muscle & 2 & BE (2002) & December & \\
\hline & Bone & 2 & BE (2002) & December & \\
\hline & Liver & 2 & BE (2002) & December & \\
\hline \multirow{3}{*}{$\begin{array}{l}\text { Road Kill at } \\
\text { Onsite Location } \\
\text { (d) }\end{array}$} & Mule Deer/Elk & & & & \multirow{3}{*}{$\begin{array}{l}\text { Gamma Scan } \\
{ }^{90} \mathrm{Sr}\end{array}$} \\
\hline & Muscle & 10 & BE (2002) & As Available & \\
\hline & Bone & 10 & BE (2002) & As Available & \\
\hline \multirow[t]{4}{*}{ Background } & Mule Deer & & & & \\
\hline & Muscle & 2 & BE (2002) & October & \multirow{3}{*}{$\begin{array}{l}\text { Gamma Scan, } \mathrm{DOH}^{(\mathrm{c})} \\
{ }^{90} \mathrm{Sr}, \mathrm{DOH}^{(\mathrm{c})} \\
\mathrm{Pu}\end{array}$} \\
\hline & Bone & 2 & BE (2002) & October & \\
\hline & Liver & 2 & BE (2002) & October & \\
\hline
\end{tabular}

(a) Refer to Figure 3.2, 2002 Wildlife Sampling Locations.

(b) Samples are collected in 2002 according to their specified frequency unless otherwise noted.

(c) One cosample provided to the Washington State Department of Health.

(d) As available, according to location. 


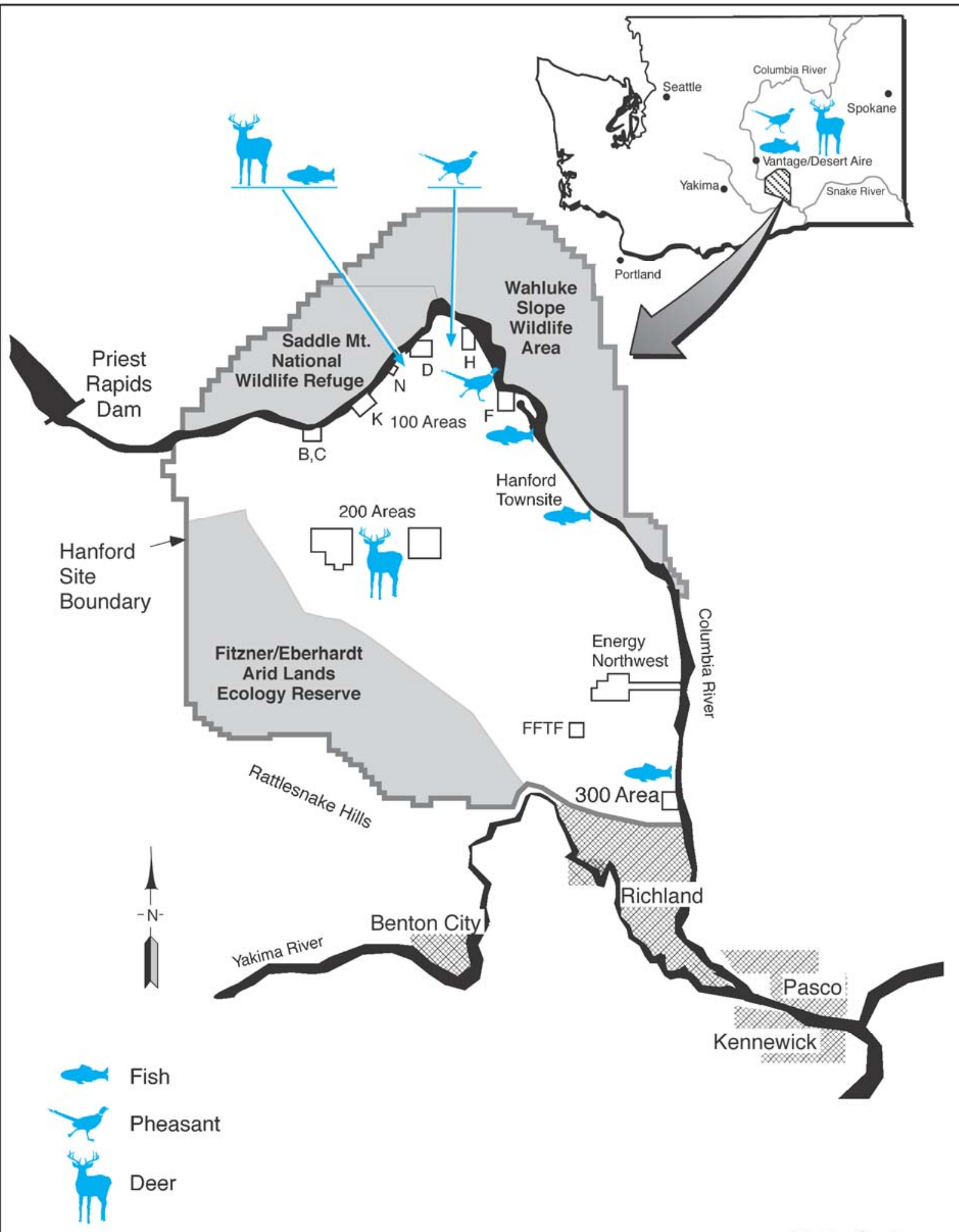

Not to Scale

G01120042.4

Figure 3.2. 2002 Wildlife Sampling Locations 


\subsection{SOIL AND VEGETATION}

\subsection{SOIL}

\begin{tabular}{|c|c|}
\hline Location & $\underline{\text { Frequency }}^{(\mathrm{a})}$ \\
\hline 100 K Area & 3 to 5 yrs \\
\hline NE of $100 \mathrm{~N}$ Area & 3 to 5 yrs \\
\hline E of 100 N Area & 3 to 5 yrs \\
\hline 100N Shore Above HGP & 3 to 5 yrs \\
\hline 100N Spring Shoreline & 3 to 5 yrs \\
\hline Above 100D Pumphouse & 3 to $5 \mathrm{yrs}$ \\
\hline 100 Area Fire Stat & 3 to $5 \mathrm{yrs}$ \\
\hline 200 ENC & 3 to 5 yrs \\
\hline E of $200 \mathrm{E}$ & 3 to 5 yrs \\
\hline 200 ESE & 3 to 5 yrs \\
\hline S of $200 \mathrm{E}$ & 3 to $5 \mathrm{yrs}$ \\
\hline SW of B/C Cribs & 3 to $5 \mathrm{yrs}$ \\
\hline E of 200 W Gate & 3 to 5 yrs \\
\hline S of $200 \mathrm{~W}$ & 3 to 5 yrs \\
\hline Rattlesnake Springs & 3 to 5 yrs \\
\hline Yakima Barricade & 3 to 5 yrs \\
\hline $400 \mathrm{E}$ & 3 to 5 yrs \\
\hline SE Side of FFTF & 3 to 5 yrs \\
\hline North of 300 Area & 3 to 5 yrs \\
\hline South of 300 Area & 3 to 5 yrs \\
\hline Hanford Townsite & 3 to 5 yrs \\
\hline Wye Barricade & 3 to 5 yrs \\
\hline Prosser Barricade & 3 to $5 \mathrm{yrs}$ \\
\hline ALE Field Lab & 3 to $5 \mathrm{yrs}$ \\
\hline N End Vernita Bridge & 3 to $5 \mathrm{yrs}$ \\
\hline Wahluke Slope & 3 to $5 \mathrm{yrs}$ \\
\hline Berg Ranch & 3 to 5 yrs \\
\hline Ringold Area & 3 to 5 yrs \\
\hline W End of Fir Road & 3 to 5 yrs \\
\hline Taylor Flats No. 2 & 3 to 5 yrs \\
\hline Sagemoor Farm & 3 to 5 yrs \\
\hline Byers Landing & 3 to 5 yrs \\
\hline Riverview-Harris & 3 to 5 yrs \\
\hline Benton City & 3 to 5 yrs \\
\hline Sunnyside & 3 to 5 yrs \\
\hline McNary Dam & 3 to 5 yrs \\
\hline Walla Walla & 3 to 5 yrs \\
\hline Washtucna & 3 to 5 yrs \\
\hline Toppenish & 3 to 5 yrs \\
\hline
\end{tabular}

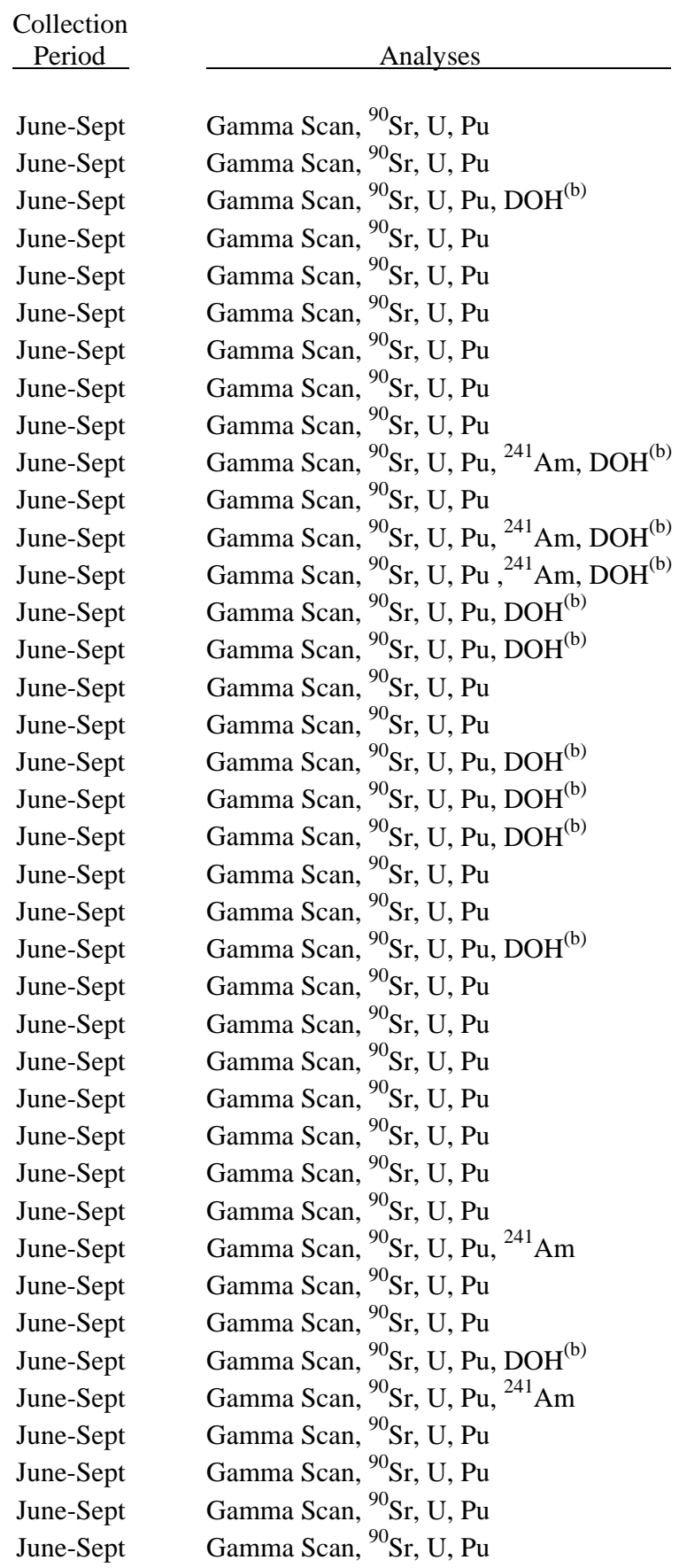

(a) Samples are collected once every 3 to 5 years and will be collected in 2004 .

(b) Cosample provided to the Washington State Department of Health. 


\subsection{VEGETATION}

\begin{tabular}{|c|c|c|c|}
\hline Location & Frequency $^{(\mathrm{a})}$ & $\begin{array}{c}\text { Collection } \\
\text { Period } \\
\end{array}$ & Analyses \\
\hline $100 \mathrm{~K}$ Area & 3 to 5 yrs & June-Sept & Gamma Scan, ${ }^{90} \mathrm{Sr}, \mathrm{U}, \mathrm{Pu}$ \\
\hline NE of $100 \mathrm{~N}$ Area & 3 to 5 yrs & June-Sept & Gamma Scan, ${ }^{90} \mathrm{Sr}, \mathrm{U}, \mathrm{Pu}$ \\
\hline E of $100 \mathrm{~N}$ Area & 3 to 5 yrs & June-Sept & Gamma Scan, ${ }^{90} \mathrm{Sr}, \mathrm{U}, \mathrm{Pu}, \mathrm{DOH}^{(\mathrm{b})}$ \\
\hline 100N Spring Shoreline & 3 to 5 yrs & June-Sept & Gamma Scan, ${ }^{90} \mathrm{Sr}, \mathrm{U}, \mathrm{Pu}$ \\
\hline E of $200 \mathrm{~W}$ Gate & 3 to 5 yrs & June-Sept & Gamma Scan, ${ }^{90} \mathrm{Sr}, \mathrm{U}, \mathrm{Pu}$ \\
\hline 300 Area Shoreline & 3 to 5 yrs & June-Sept & Gamma Scan, ${ }^{90} \mathrm{Sr}, \mathrm{U}, \mathrm{Pu}, \mathrm{DOH}^{(\mathrm{b})}$ \\
\hline Hanford Townsite & 3 to 5 yrs & June-Sept & Gamma Scan, ${ }^{90} \mathrm{Sr}, \mathrm{U}, \mathrm{Pu}$ \\
\hline Hanford Twnsite HRM28 & 3 to 5 yrs & June-Sept & Gamma Scan, ${ }^{90} \mathrm{Sr}, \mathrm{U}, \mathrm{Pu},{ }^{99} \mathrm{Tc}, \mathrm{DOH}^{(\mathrm{b})}$ \\
\hline Ringold Area & 3 to 5 yrs & June-Sept & Gamma Scan, ${ }^{90} \mathrm{Sr}, \mathrm{U}, \mathrm{Pu}$ \\
\hline Sagemoor Farm & 3 to 5 yrs & June-Sept & Gamma Scan, ${ }^{90} \mathrm{Sr}, \mathrm{U}, \mathrm{Pu}$ \\
\hline Byers Landing & 3 to 5 yrs & June-Sept & Gamma Scan, ${ }^{90} \mathrm{Sr}, \mathrm{U}, \mathrm{Pu}$ \\
\hline Riverview-Harris & 3 to 5 yrs & June-Sept & Gamma Scan, ${ }^{90} \mathrm{Sr}, \mathrm{U}, \mathrm{Pu}$ \\
\hline Sunnyside & 3 to 5 yrs & June-Sept & Gamma Scan, ${ }^{90} \mathrm{Sr}, \mathrm{U}, \mathrm{Pu}$ \\
\hline Toppenish & 3 to 5 yrs & June-Sept & Gamma Scan, ${ }^{90} \mathrm{Sr}, \mathrm{U}, \mathrm{Pu}$ \\
\hline
\end{tabular}

(a) Samples are collected once every 3 to 5 years and will be collected in 2004 .

(b) Cosample provided to the Washington State Department of Health. 


\subsection{SEDIMENT}

Location $^{(\mathrm{a})}$

Onsite Pond

West Lake

$\underline{\text { River }}$

McNary Dam

McNary-OR. Side Near Dam

McNary-Wash. Side Near Dam

Priest Rapids Dam (PRD)

PRD-Grant Side Near Dam

PRD-Yakima Side Near Dam

White Bluffs Slough

100 F Slough

Hanford Slough

Richland

Springs

100-B Spring 38-3

100-K Spring 63-1

100-H Spring 145-1

100-F Spring 207-1

Hanford Spr UR 28-2

Hanford Spr DR 28-2

300 Area Spring 42-2

300 Area Spr DR 42-2
Frequency

Analyses

Q Gamma Scan ${ }^{90} \mathrm{Sr}, \mathrm{U},{ }^{99} \mathrm{Tc}$, Alpha, Beta

A Gamma Scan, ${ }^{90} \mathrm{Sr}, \mathrm{U}, \mathrm{Pu}, \mathrm{ICP}-\mathrm{u}, \mathrm{SEM} / \mathrm{AVS}, \mathrm{TOC}, \mathrm{DOH}^{(\mathrm{b})}$

A Gamma Scan, ${ }^{90} \mathrm{Sr}, \mathrm{U}, \mathrm{Pu}, \mathrm{ICP}-\mathrm{u}, \mathrm{SEM} / \mathrm{AVS}, \mathrm{TOC}, \mathrm{DOH}^{(\mathrm{b})}$

A Gamma Scan, ${ }^{90} \mathrm{Sr}, \mathrm{U}, \mathrm{Pu}, \mathrm{ICP}-\mathrm{u}, \mathrm{SEM} / \mathrm{AVS}, \mathrm{TOC}, \mathrm{DOH}^{(\mathrm{b})}$

A Gamma Scan, ${ }^{90} \mathrm{Sr}, \mathrm{U}, \mathrm{Pu}, \mathrm{ICP}-\mathrm{u}, \mathrm{SEM} / \mathrm{AVS}, \mathrm{TOC}, \mathrm{DOH}^{(\mathrm{b})}$

A Gamma Scan, ${ }^{90} \mathrm{Sr}, \mathrm{U}, \mathrm{Pu}, \mathrm{ICP}-\mathrm{u}, \mathrm{SEM} / \mathrm{AVS}, \mathrm{TOC}$

A

Gamma Scan, ${ }^{90}$ Sr, U, Pu, ICP-u, SEM/AVS, TOC, DOH ${ }^{(b)}$

A

Gamma Scan, ${ }^{90} \mathrm{Sr}, \mathrm{U}, \mathrm{Pu}, \mathrm{ICP}-\mathrm{u}, \mathrm{SEM} / \mathrm{AVS}$, TOC

A

Gamma Scan, ${ }^{90} \mathrm{Sr}, \mathrm{U}, \mathrm{Pu}, \mathrm{ICP}-\mathrm{u}, \mathrm{SEM} / \mathrm{AVS}, \mathrm{TOC}$

A

Gamma Scan, ${ }^{90} \mathrm{Sr}, \mathrm{U}, \mathrm{ICP}-\mathrm{u}$

A

Gamma Scan, ${ }^{90}$ Sr, U, ICP-u, DOH ${ }^{(b)}$

A

Gamma Scan, ${ }^{90} \mathrm{Sr}, \mathrm{U}, \mathrm{ICP}-\mathrm{u}, \mathrm{DOH}^{(\mathrm{b})}$

A

Gamma Scan, ${ }^{90}$ Sr, U, ICP-u, DOH ${ }^{(b)}$

A

Gamma Scan, ${ }^{90} \mathrm{Sr}$, U, ICP-u

A

Gamma Scan, ${ }^{90} \mathrm{Sr}, \mathrm{U}, \mathrm{ICP}-\mathrm{u}, \mathrm{DOH}^{(\mathrm{b})}$

A Gamma Scan, ${ }^{90} \mathrm{Sr}, \mathrm{U}, \mathrm{ICP}-\mathrm{u}$

A Gamma Scan, ${ }^{90}$ Sr, U, ICP-u

(a) Refer to Figure 5.1, 2002 Sediment Sampling Locations. UR and DR referenced to upriver and downriver.

(b) Cosample provided to the Washington State Department of Health. 


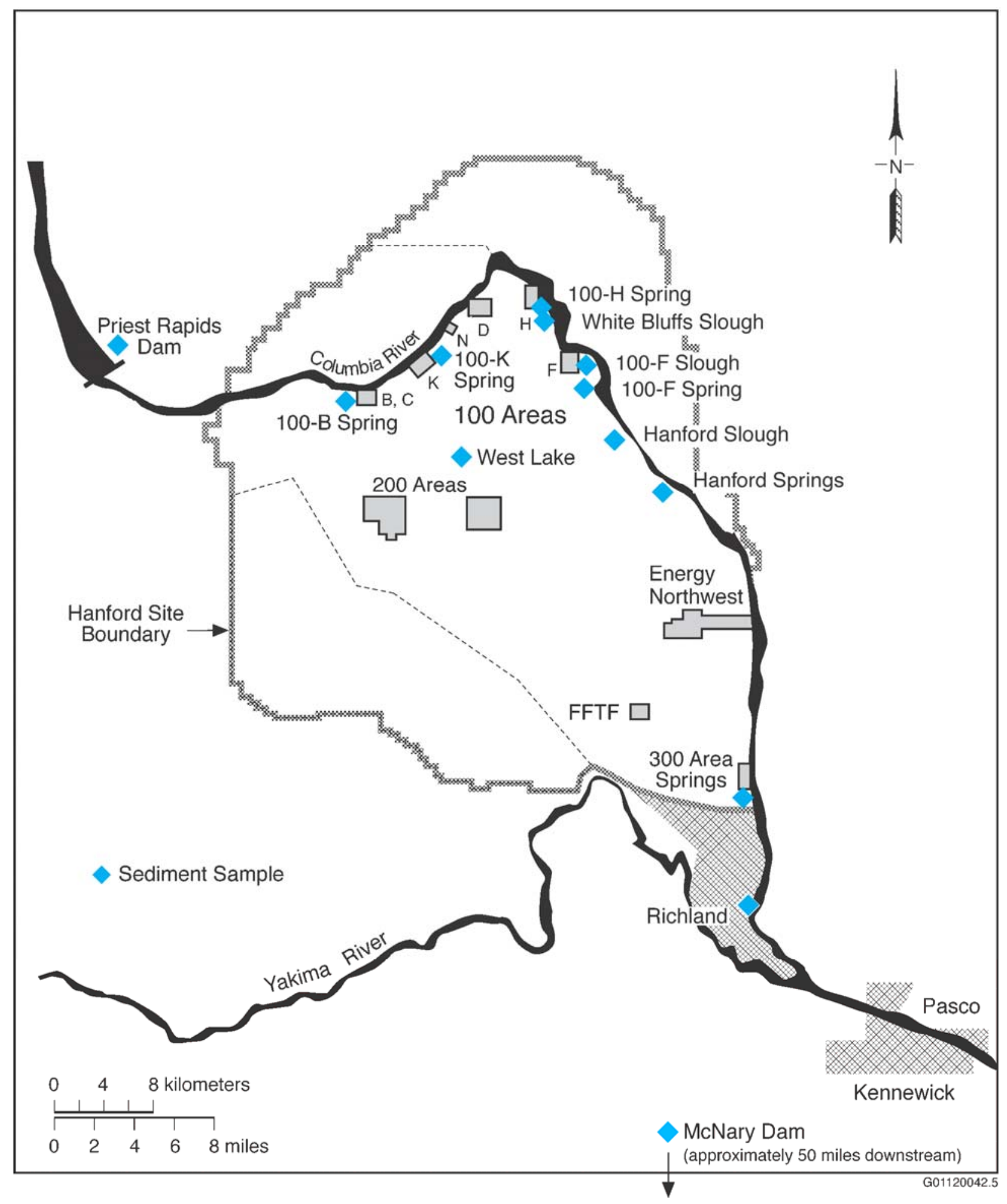

Figure 5.1. 2002 Sediment Sampling Locations 


\subsection{EXTERNAL RADIATION}

\subsection{THERMOLUMINESCENT DOSIMETERS (TLDS)}

\subsubsection{Terrestrial Locations}

\begin{tabular}{|c|c|c|}
\hline Location & $\begin{array}{c}\text { Location } \\
\text { Number } \\
\end{array}$ & Frequen \\
\hline \multicolumn{3}{|l|}{ Onsite $^{(\mathrm{a})}$} \\
\hline 100 B Reactor Museum & 1 & Q \\
\hline 100 K Area $^{(\mathrm{c})}$ & 2 & $\mathrm{Q}$ \\
\hline 100 D Area $^{(c)}$ & 3 & $\mathrm{Q}$ \\
\hline 100 F Met Tower ${ }^{(c)}$ & 4 & $\mathrm{Q}$ \\
\hline Hanford Townsite ${ }^{(c)}$ & 5 & $\mathrm{Q}$ \\
\hline West Lake & 6 & $\mathrm{Q}$ \\
\hline$N$ of $200 E^{(c)}$ & 7 & $\mathrm{Q}$ \\
\hline B Pond ${ }^{(c)}$ & 8 & $\mathrm{Q}$ \\
\hline$E$ of $200 E^{(c)}$ & 9 & $\mathrm{Q}$ \\
\hline $200 \mathrm{ESE}^{(\mathrm{c})}$ & 10 & $\mathrm{Q}$ \\
\hline S of $200 \mathrm{E}^{(\mathrm{c})}$ & 11 & $\mathrm{Q}$ \\
\hline 200 Tel. Exchange ${ }^{(\mathrm{c})}$ & 12 & $\mathrm{Q}$ \\
\hline SW of B/C Cribs ${ }^{(c)}$ & 13 & $\mathrm{Q}$ \\
\hline $200 \mathrm{~W} \mathrm{SE}^{(\mathrm{c})}$ & 14 & $\mathrm{Q}$ \\
\hline Army Loop Camp ${ }^{(c)}$ & 15 & $\mathrm{Q}$ \\
\hline 3705 Bldg. 300 Area & 16 & Q \\
\hline 313 Bldg. & 17 & $\mathrm{Q}$ \\
\hline 300 Water Intake $e^{(c)}$ & 18 & $\mathrm{Q}$ \\
\hline 300 Southwest Gate & 19 & Q \\
\hline 300 South Gate ${ }^{(\mathrm{c})}$ & 20 & $\mathrm{Q}$ \\
\hline 300 Trench $^{(c)}$ & 21 & $\mathrm{Q}$ \\
\hline $300 \mathrm{NE}^{(\mathrm{c})}$ & 22 & $\mathrm{Q}$ \\
\hline $400 \mathrm{E}^{(\mathrm{c})}$ & 23 & $\mathrm{Q}$ \\
\hline $400 \mathrm{~W}^{(\mathrm{c})}$ & 24 & $\mathrm{Q}$ \\
\hline $400 S^{(c)}$ & 25 & $\mathrm{Q}$ \\
\hline $400 \mathrm{~N}^{(\mathrm{c})}$ & 26 & $\mathrm{Q}$ \\
\hline US Ecology NE Corner & 27 & $\mathrm{Q}$ \\
\hline US Ecology SE Corner & 28 & $\mathrm{Q}$ \\
\hline US Ecology NW Corner & 29 & Q \\
\hline US Ecology SW Corner & 30 & Q \\
\hline Wye Barricade ${ }^{(c)}$ & 31 & $\mathrm{Q}$ \\
\hline WPPSS 1; S of WNP 2 & 32 & $\mathrm{Q}$ \\
\hline LIGO & 33 & Q \\
\hline
\end{tabular}

Perimeter $^{(\mathrm{d})}$

Ringold Met Tower
W End of Fir Road $^{(\mathrm{c})}$
Dogwood Met Tower $^{(\mathrm{c})}$
Byers Landing $^{(\mathrm{c})}$
Battelle Complex
WPPSS 4; WPS Warehse
Horn Rapids Substa $^{(\mathrm{c})}$

$\begin{array}{ll}1 & \mathrm{Q} \\ 2 & \mathrm{Q} \\ 3 & \mathrm{Q} \\ 4 & \mathrm{Q} \\ 5 & \mathrm{Q} \\ 6 & \mathrm{Q} \\ 7 & \mathrm{Q}\end{array}$

\begin{abstract}
Ambient Dose, $\mathrm{DOH}^{(\mathrm{b})}$
Ambient Dose

Ambient Dose

Ambient Dose

Ambient Dose

Ambient Dose

Ambient Dose, $\mathrm{DOH}^{(\mathrm{b})}$

Ambient Dose

Ambient Dose, $\mathrm{DOH}^{(\mathrm{b})}$

Ambient Dose

Ambient Dose

Ambient Dose

Ambient Dose

Ambient Dose

Ambient Dose

Ambient Dose

Ambient Dose, $\mathrm{DOH}^{(\mathrm{b})}$

Ambient Dose

Ambient Dose

Ambient Dose

Ambient Dose

Ambient Dose

Ambient Dose

Ambient Dose

Ambient Dose

Ambient Dose

Ambient Dose, $\mathrm{DOH}^{(\mathrm{b})}$

Ambient Dose, $\mathrm{DOH}^{(\mathrm{b})}$

Ambient Dose, $\mathrm{DOH}^{(\mathrm{b})}$

Ambient Dose, $\mathrm{DOH}^{(\mathrm{b})}$

Ambient Dose, $\mathrm{DOH}^{(\mathrm{b})}$

Ambient Dose, $\mathrm{DOH}^{(\mathrm{b})}$

Ambient Dose, $\mathrm{DOH}^{(\mathrm{b})}$
\end{abstract}

Ambient Dose

Ambient Dose, DOH ${ }^{(b)}$

Ambient Dose

Ambient Dose, $\mathrm{DOH}^{(\mathrm{b})}$

Ambient Dose

Ambient Dose, $\mathrm{DOH}^{(\mathrm{b})}$

Ambient Dose, $\mathrm{DOH}^{(\mathrm{b})}$ 


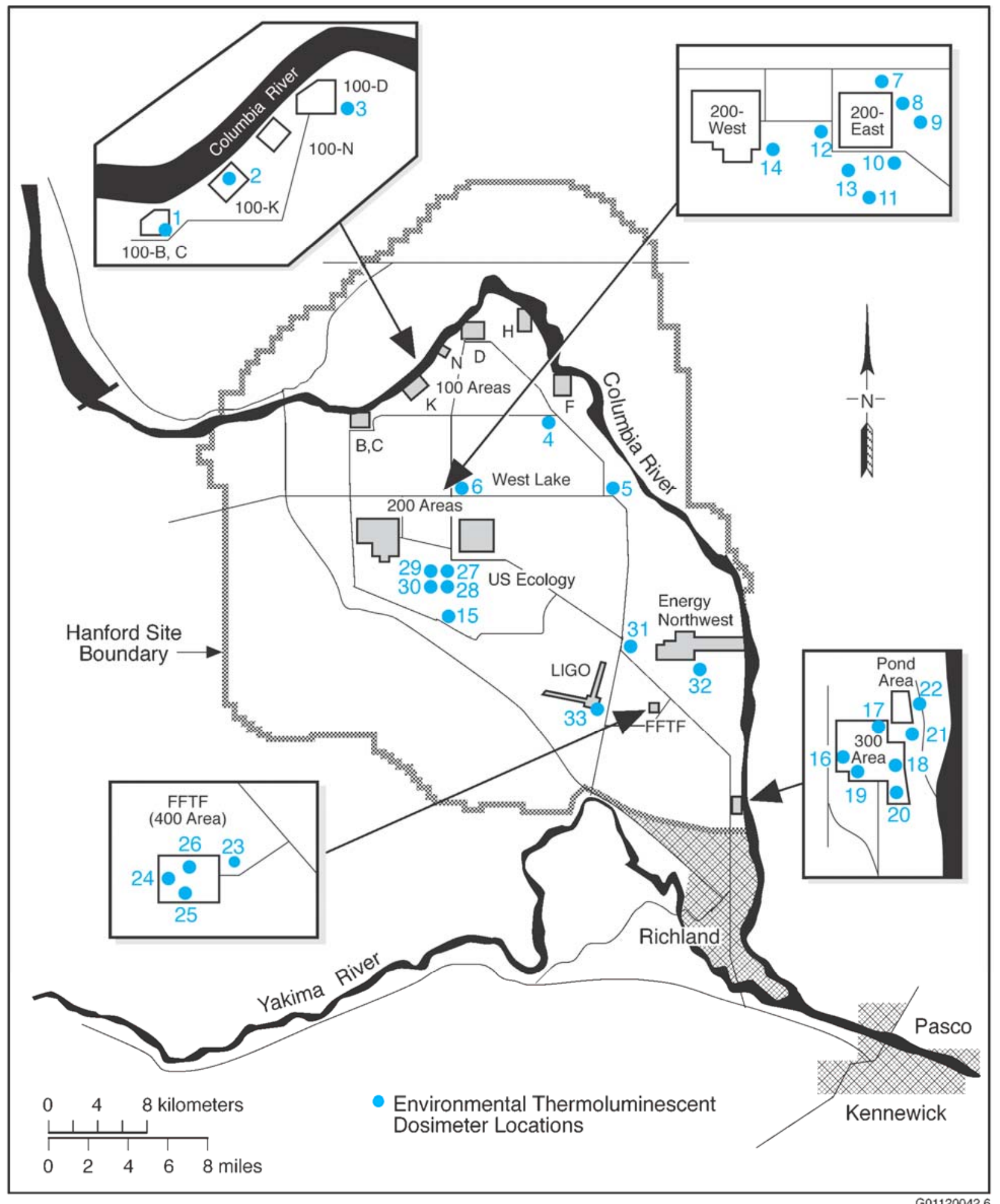

Figure 6.1. 2002 Thermoluminescent Dosimeter (TLD) Locations on the Hanford Site 


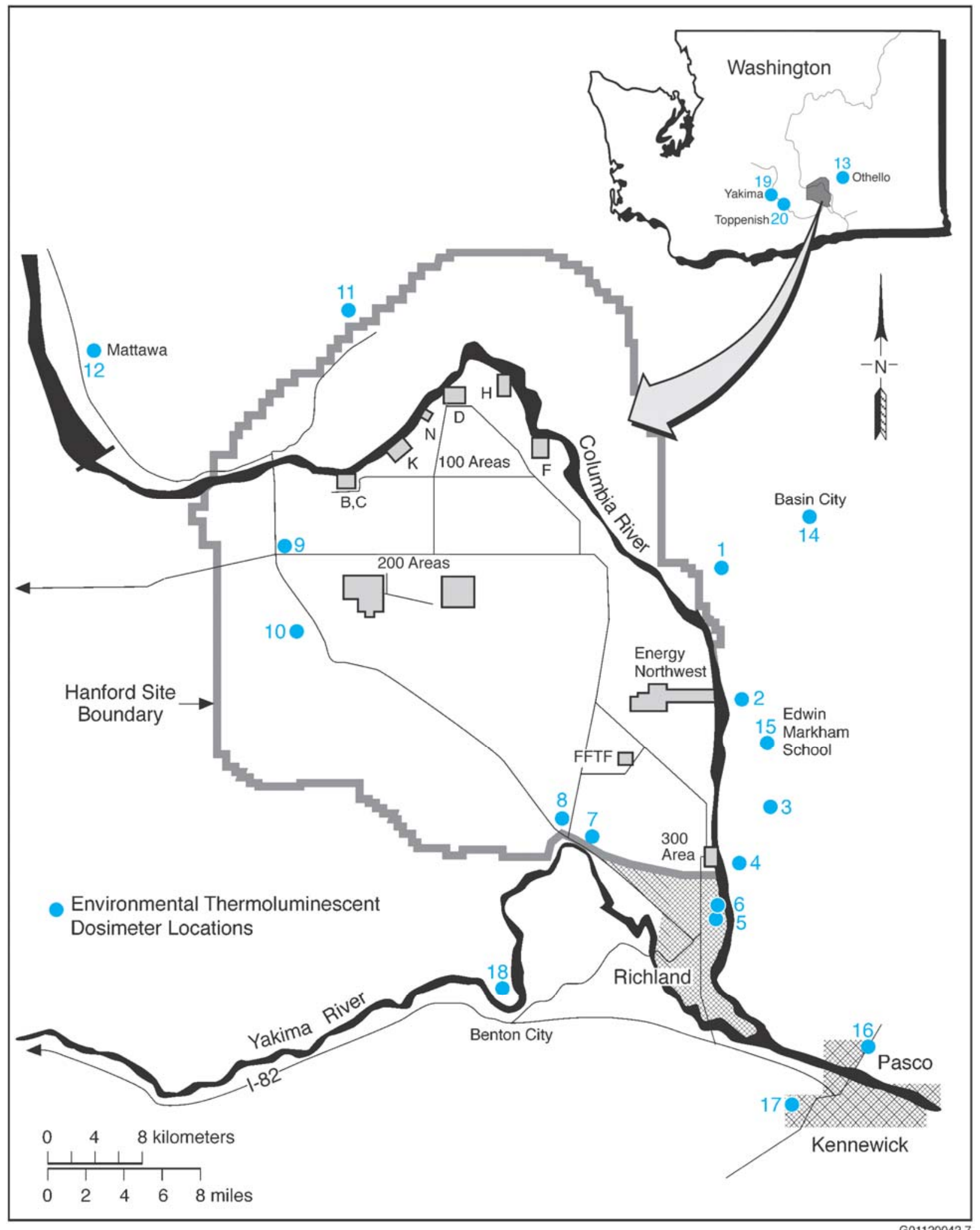

Figure 6.2. 2002 Thermoluminescent Dosimeter (TLD) Locations for Perimeter, Community, and Distant Sites 


\subsubsection{Columbia River Shoreline Locations}

\begin{tabular}{l}
\multicolumn{1}{c}{ Location $^{(\mathrm{a})}$} \\
\hline \\
S End Vernita Bridge \\
Above 100 B Area \\
Below 100 B Ret Basin \\
Above 1K Boat Ramp \\
Below 100N Outfall \\
Above Tip 100N Berm \\
100 N Trench Spring \\
Below 100 D Area \\
100-D Island \\
100 H Area \\
Lo End Locke Isl \\
White Bluffs Fy Lnd. \\
White Bluffs Slough \\
Below 100 F \\
100 F Floodplain \\
Hanford Slough \\
Hanf Powerline Xing \\
Hanford RR Track \\
Savage Isl Slough \\
Ringold Island \\
Powerline Crossing \\
S End Wooded Island \\
Islnd Above 300 Area \\
Island Near 300 Area \\
Port of Benton-River \\
N. Richland \\
Isl DS Bateman Isl \\
\end{tabular}

\begin{tabular}{l}
$\begin{array}{c}\text { Location } \\
\text { Number }\end{array}$ \\
\hline 1 \\
2 \\
3 \\
4 \\
5 \\
6 \\
7 \\
8 \\
9 \\
10 \\
11 \\
12 \\
13 \\
14 \\
15 \\
16 \\
17 \\
18 \\
19 \\
20 \\
21 \\
22 \\
23 \\
24 \\
25 \\
26 \\
27
\end{tabular}

\begin{tabular}{cc} 
Frequency & Measurement \\
\cline { 2 - 2 } Q & \\
Q & Ambient Dose \\
Q & Ambient Dose \\
Q & Ambient Dose \\
Q & Ambient Dose \\
Q & Ambient Dose \\
Q & Ambient Dose \\
Q & Ambient Dose \\
Q & Ambient Dose \\
Q & Ambient Dose \\
Q & Ambient Dose \\
Q & Ambient Dose \\
Q & Ambient Dose \\
Q & Ambient Dose \\
Q & Ambient Dose \\
Q & Ambient Dose \\
Q & Ambient Dose \\
Q & Ambient Dose \\
Q & Ambient Dose \\
Q & Ambient Dose \\
Q & Ambient Dose \\
Q & Ambient Dose \\
Q & Ambient Dose \\
Q & Ambient Dose \\
Q & Ambient Dose \\
Q & Ambient Dose \\
Q & Ambient Dose
\end{tabular}

$\underline{\text { Instrument }}$

(a) Refer to Figure 6.3, 2002 Thermoluminescent Dosimeter (TLD) Locations on the Hanford Reach of the Columbia River.

(b) Collocated with air sampling station.

(c) PIC located at Leslie Groves-Rchlnd air sampling station.

\subsection{COLUMBIA RIVER SHORELINE RADIATION SURVEYS}

\begin{tabular}{|c|c|c|c|c|}
\hline Location $^{(\mathrm{a})}$ & $\begin{array}{l}\text { Location } \\
\text { Number }\end{array}$ & $\underline{\text { Frequency }}$ & Measurement & Instrument \\
\hline S End Vernita Bridge & 1 & Q & Exposure, Surface contamination & BICRON, GM \\
\hline Above $1 \mathrm{~K}$ Boat Ramp & 4 & $\mathrm{Q}$ & Exposure, Surface contamination & BICRON, GM \\
\hline Below 100N Outfall & 5 & Q & Exposure, Surface contamination & BICRON, GM \\
\hline Above Tip 100N Berm & 6 & $\mathrm{Q}$ & Exposure, Surface contamination & BICRON, GM \\
\hline 100 N Trench Spring & 7 & $\mathrm{Q}$ & Exposure, Surface contamination & BICRON, GM \\
\hline 100-D Island & 9 & $\mathrm{Q}$ & Exposure, Surface contamination & BICRON, GM \\
\hline Lo End Locke Isl & 11 & $\mathrm{Q}$ & Exposure, Surface contamination & BICRON, GM \\
\hline White Bluffs Fy Lnd. & 12 & $\mathrm{Q}$ & Exposure, Surface contamination & BICRON, GM \\
\hline Below $100 \mathrm{~F}$ & 14 & $\mathrm{Q}$ & Exposure, Surface contamination & BICRON, GM \\
\hline Hanf Powerline Xing & 17 & $\mathrm{Q}$ & Exposure, Surface contamination & BICRON, GM \\
\hline Hanford RR Track & 18 & $\mathrm{Q}$ & Exposure, Surface contamination & BICRON, GM \\
\hline Ringold Island & 20 & $\mathrm{Q}$ & Exposure, Surface contamination & BICRON, GM \\
\hline Powerline Crossing & 21 & $\mathrm{Q}$ & Exposure, Surface contamination & BICRON, GM \\
\hline Islnd Above 300 Area & 23 & $\mathrm{Q}$ & Exposure, Surface contamination & BICRON, GM \\
\hline
\end{tabular}

(a) Refer to Figure 6.3, 2002 Thermoluminescent Dosimeter (TLD) Locations on the Hanford Reach of the Columbia River. 


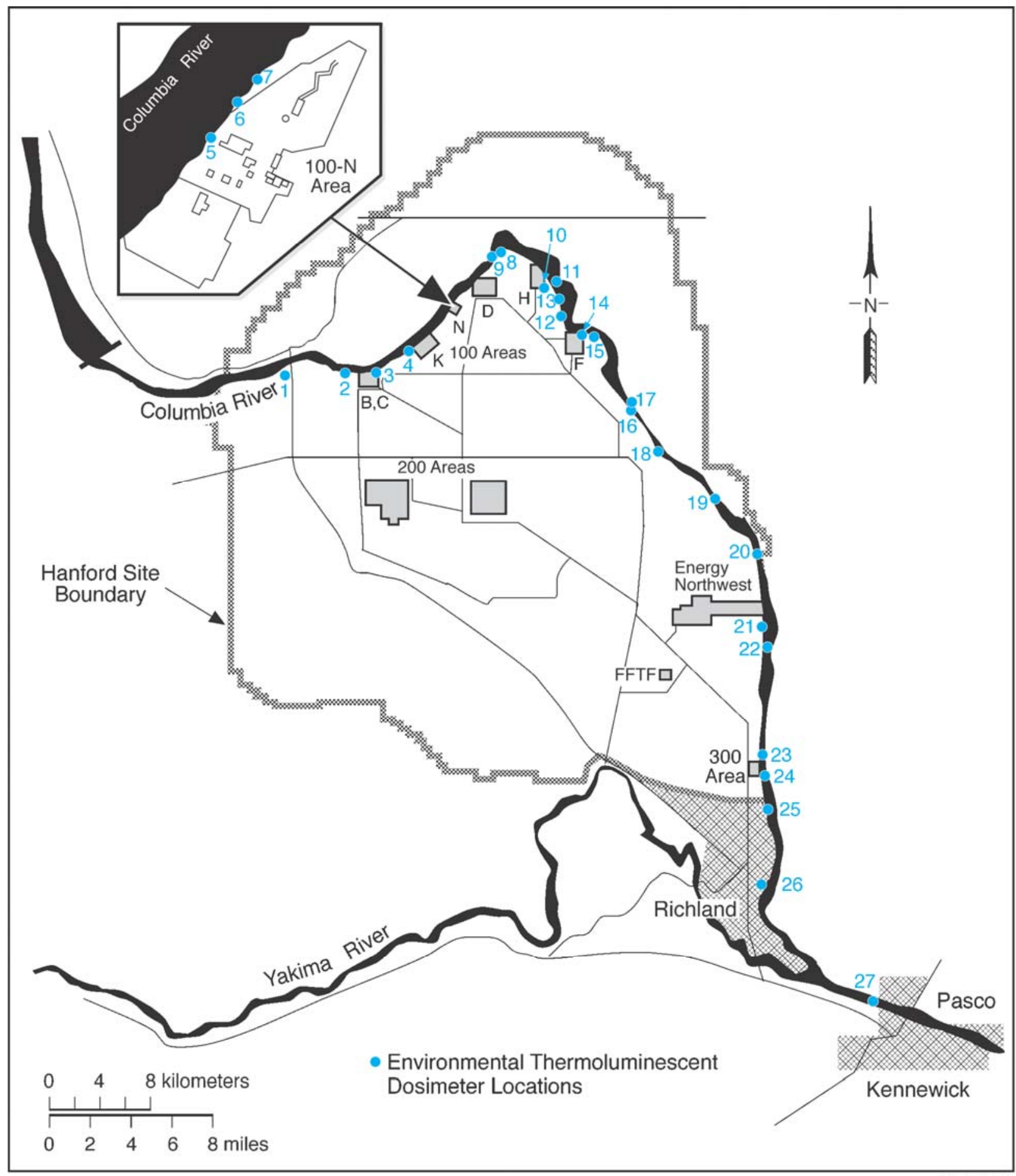

G01120042.8

Figure 6.3. 2002 Thermoluminescent Dosimeter (TLD) Locations on the Hanford Reach of the Columbia River 
PNNL-13749

\section{DISTRIBUTION}

No. of

$\underline{\text { Copies }}$

\section{OFFSITE (26)}

2 L. Albin

Division of Radiation Protection

Washington State Dept. of Health

P.O. Box 47827

Olympia, WA 98504-7827

R. Buck, Jr.

Wanapum Indian Band

P.O. Box 878

Ephrata, WA 98823

A. T. Cooper

Kennewick, WA 99337

2 A. Danielson

Washington State Dept. of Health

5508 Englewood Avenue

Yakima, WA 98908

R. Gay

Environmental Science and Technology

Program

Confederated Tribes of the Umatilla Indian

Reservation

P.O. Box 638

Pendleton, OR 97801

M. L. Goldstein

U.S. Environmental Protection Agency

P.O. Box 550, MS B5-01

Richland, WA 99352

R. E. Jaquish

1232 Vintage Avenue

Richland, WA 99352

R. Jim

Environmental Restoration/Waste Management

Program

Yakama Nation

2808 Main Street

Union Gap, WA 98903
No. of

Copies

D. Landeen

Environmental Restoration/Waste Management Program

Nez Perce Tribe

P.O. Box 365

Lapwai, ID 83540

S. Langford

Division of Radiation Protection

Washington State Dept. of Health

P.O. Box 47827

Olympia, WA 98504-7827

D. McBaugh, Head, MS-7827

Environmental Radiation Section

Division of Radiation Protection

Washington State Dept. of Health

P.O. Box 47827

Olympia, WA 98504-7827

J. E. McDonald, MS-1025

Environmental Scientist

Energy Northwest

P.O. Box 968

Richland, WA 99352

C. Palmer

Department of Natural Resources

Yakama Nation

P.O. Box 151

Toppenish, WA 98948

D. Powaukee

Environmental Restoration/Waste Management Program

Nez Perce Tribe

P.O. Box 365

Lapwai, ID 83540

J. Richards

Confederated Tribes of the

Umatilla Indian Reservation

P.O. Box 638

Pendleton, OR 97801

Distr. 1 
No. of

Copies

L. Seelatsee

Wanapum34

Grant County P.U.D.

30 "C" Street, S.W.

P.O. Box 878

Ephrata, WA 98823

2 D. R. Sherwood

U.S. Environmental Protection Agency

P.O. Box 550, MS B5-01

Richland, WA 99352

P. Sobotta

Environmental Restoration/Waste Management

Program

Nez Perce Tribe

P.O. Box 365

Lapwai, ID 83540

S. Van Verst

Division of Radiation Protection

Washington State Dept. of Health

P.O. Box 47827

Olympia, WA 98504-7827

J. R. Wilkinson

Special Science and Resource Program

Department of Natural Resources

P.O. Box 638

Pendleton, OR 97801

3 M. A. Wilson

Washington State Dept. of Ecology

1315 4th Avenue

Kennewick, WA 99336

\section{ONSITE (66)}

\section{DOE Richland Operations}

E. M. Bowers

A2-15

J. B. Hall

A2-15

R. D. Hildebrand

M. Thompson

A. C. Tortoso
D. C. Ward (5)

S. H. Wisness

A2-15

J. Zeisloft

A2-15

DOE Public Reading Room

A2-15

H2-53

Bechtel Hanford, Inc.

K. A. Gano

H0-23

Fluor Hanford

A.R. Johnson

H5-26

CH2M Hill Hanford Group, Inc.

R.W. Ovink

H9-01

4 Duratek Federal Services Northwest

J. J. Dorian

H1-11

S. M. McKinney

H1-11

R. M. Mitchell

H1-11

C. J. Perkins

H1-11

46 Pacific Northwest National Laboratory

E. J. Antonio

K3-54

L. E. Bisping (30)

K6-75

R. L. Dirkes

K6-75

P. E. Dresel

K6-96

B. G. Fritz

K6-75

R. W. Fulton

K6-75

R. W. Hanf, Jr.

K6-75

S. E. Huneycutt

P7-02

B. A. Napier

K3-54

B. E. Opitz

K6-75

G. W. Patton

K6-75

R.E. Peterson

K6-96

T. M. Poston

K6-75

B. A. Rathbone

P7-02

G. A. Stoetzel

P7-78

B. L. Tiller

K6-85

Historical File-T. M. Poston K6-75

Distr. 2 